\title{
Classical and Quantum Statistical Mechanics in One and Two Dimensions: Two-Component Yukawa - and Coulomb Systems
}

\author{
Jürg Fröhlich \\ Department of Mathematics, Princeton University, Princeton, N. J. 08540, USA
}

\begin{abstract}
We estimate the canonical and grand canonical partition function in a finite volume and prove stability and existence of the thermodynamic limit for the pressure of two component classical and quantum systems of particles with charge $\pm \varepsilon$ interacting via two body Yukawa - or Coulomb forces. In the case of Coulomb forces we require neutrality. For the classical system in two dimensions there exists a critical temperature $T_{c}$ at and below which the system collapses. For the classical Yukawa system the correlation functions exist for arbitrary fugacity and the general structure of the pure phases can be analyzed completely.
\end{abstract}

\section{Introduction}

a) Definition of the Problem; Connections to Euclidean Field Theory

In this paper we construct the thermodynamic limit of the pressure for systems of classical point particles in two space dimensions with charge $\pm \varepsilon$ interacting via Yukawa - or Coulomb - two body forces (or forces which can be "dominated" by these). The same result for the analogous systems in one dimension [6] and for the corresponding quantum systems in (one and) two dimensions then follow as rather simple corollaries, at least if one borrows certain estimates and techniques from [19] for the analysis of the quantum systems.

The two dimensional classical systems we are considering are not $H$-stable [26], the classical Hamilton function is not bounded below, and we should therefore not be surprised that there exists a critical temperature $T_{c}$ depending on the strength of the forces, i.e. the charge $\varepsilon$, at and below which these classical systems collapse. This is due to the lack of a sufficiently strong centrifugal barrier below $T_{c}$. Also notice that the Coulomb potential in one and two dimensions is extremely long range $(\sim r, \log r$, respectively, where $r$ is the distance between two charges). It is therefore not tempered. If the total charge is 0 there is screening, and the long range character of the Coulomb forces disappears. That is why we only consider neutral Coulomb systems in this paper. Results on the classical 
Coulomb system in a finite volume have previously been obtained in [4]. Their estimates are essential for this paper.

Our approach towards a solution of these problems consists in viewing a system of classical point particles in two space dimensions with charge $\pm \varepsilon$ interacting via Yukawa - or Coulomb forces as a particular model of Euclidean field theory (EFT; see $[7,28]$ of one neutral, scalar Bose field in two space-time dimensions $[6,1]$. (This paper is actually motivated by an analysis of a two dimensional quantum field model.) This permits us to combine estimates from statistical mechanics with estimates from Euclidean field theory. The correct dependence of the grand canonical partition function on the volume, to mention one example, is obtained by using EFT methods.

Let $s$ denote the number of space - and $d$ the number of space-time dimensions. Let CSM be an abbreviation for "classical statistical mechanics of point particles interacting via two-body-forces". The following isomorphisms have recently attracted a great deal of attention (the reader not very interested in or not familiar with quantum field theory should ignore what is described under $C, \tilde{C}$, or even under $B, \tilde{B}$, below):

$$
A
$$

$B$

C

$$
\left(\begin{array}{l}
\text { CSM, Coulomb } \\
\text { forces, } s=2
\end{array}\right) \cong\left(\begin{array}{l}
\square \Phi(x, t) \\
=\frac{z}{\sqrt{\beta \varepsilon}}: \sin \sqrt{\beta} \varepsilon \Phi(x, t): \\
d=2
\end{array}\right) \cong\left(\begin{array}{l}
\text { Massive Thirring } \\
\text { model }(+ \text { Yukawa } \\
\text { interaction }), d=2
\end{array}\right)
$$

Here $\square$ is the two dimensional d'Alembertian, $\Phi$ is a neutral, scalar quantum field of bare mass $0, z$ is a coupling constant, and $0<\beta \varepsilon^{2}<4 \pi$, :- : denotes Wick ordering. The isomorphism $I_{1}$ identifies $\beta$ with the inverse temperature, $\varepsilon$ with the charge, $z$ with the fugacity; in CSM language :- : corresponds to ignoring selfinteractions of particles. The isomorphism $I_{1}$ makes powerful EFT techniques applicable in $A$ and vice versa.

The massive Thirring model (+Yukawa interaction) is described in [2] and refs. given there. (See also $[18,20]$.) Its existence is established in $[8,9]$. It is a Fermion (-Boson) model with current-current (+Yukawa) interaction. The isomorphism $I_{2}$ has been discovered by Coleman [2]. Results proven in this paper for $A$ have turned out to be essential for an existence proof of the field theories defined in $B$ and $C$ [8].

CSM with Yukawa forces has turned out to be isomorphic to the following quantum field models:

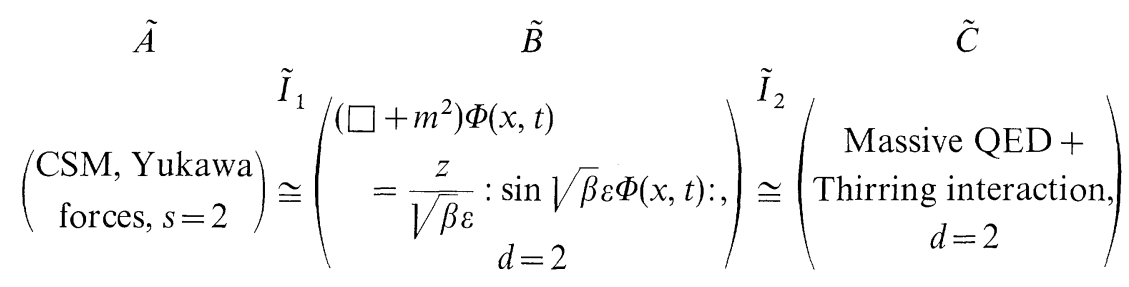


Here $\Phi$ is a neutral, scalar quantum field of bare mass $m>0 . \tilde{I}_{1}$ yields the same identifications between $\tilde{A}$ and $\tilde{B}$ as $I_{1}$ between $A$ and $B$ if one replaces Coulombby Yukawa - potentials.

Massive Quantum Electrodynamics (QED) in two space-time dimensions is a pure Fermion quantum field model with a one dimensional Coulomb force producing a self-coupling of the charge density. The Thirring interaction is a current-current interaction. The construction of the isomorphism $\tilde{I}_{2}$ can essentially be reduced to the one of $I_{2}$ (see [2]) combined with the Schwinger mechanism [27] and is given in [9]. It might be a rather easy and pleasing occupation to find many more isomorphisms of the type of $I_{2}, \tilde{I}_{2}$ in one dimensional field theoretic models of solid state physics; see $[18,20]$. In [9] we propose a "machine" which generates such isomorphisms systematically.

The critical temperature $T_{c}$ at and below which the CSM systems $A$ and $\tilde{A}$ collapse is given by

$$
T_{c}=\frac{\varepsilon^{2}}{4 \pi k}, \quad \text { or } \quad \beta \varepsilon^{2}=4 \pi,
$$

where $k$ is the Boltzmann constant. This collapse can be interpreted as a complete formation of pairs of oppositely charged particles. After a suitable renormalization of the average energy per particle the system at and below $T_{c}$ is identical to a free, classical gas of neutral particles (the pairs) and $T_{c}$ becomes a critical point.

To the collapse catastrophe there corresponds the fact that for $\beta \varepsilon^{2} \geqq 4 \pi$ the field theories described in $B, \tilde{B}, C$ and $\tilde{C}$ do no longer exist, at least without ultraviolet renormalizations; see $[8,9]$.

The classical correlation functions for the CSM system defined in $\tilde{A}$ (and the Euclidean Green's or Schwinger functions of the field theories defined in $\tilde{B}$ and $\tilde{C}$ ) can be constructed for small $\left|z / m^{2}\right|$ and for arbitrary, real $z$, provided $\beta \varepsilon^{2}<\frac{16}{\pi}$. They are analytic in $z$ in some neighborhood of $z=0$ which implies absence of phase transitions for small fugacity. The space-translation invariant pure phase correlation functions are Euclidean invariant and have the cluster property, even in the multiple phase region.

\section{b) The Physics of the Two Dimensional Coulomb System}

Statistical mechanics of the two dimensional Coulomb system is the theory of inifinitely long, parallel, charged wires - or wires conducting a current $\pm \varepsilon^{1}-$ in thermal equilibrium. To a reasonable approximation such systems exist in nature. E.g. the strongly magnetized, real plasma in thermal equilibrium can approximately be described by a two dimensional, neutral Coulomb system in the grand canonical ensemble; see [4]. For such a system we expect Debye-screening so that the classical Ursell functions decay presumably exponentially in the separation of two of their arguments. In field theory language this would imply that the energy - momentum spectrum of the field theories $B, C$ has a mass gap. The classical neutral Coulomb system has a simple scaling behaviour which we use to

1 Or e.g. vortices. 
rigorously derive explicit expressions for the pressure and the correlation length as functions of the fugacity $z$ and to provide a complete proof of the equation of state

$$
p=\frac{\varrho}{\beta}\left(1-\frac{\beta \varepsilon^{2}}{8 \pi}\right)
$$

found earlier in [21]. (Here $\varrho$ is the density.) Our results prove absence of phase transitions in the classical, neutral Coulomb gas at a fixed temperature $T>T_{c}$, as one varies the density of the gas.

The quantum theory of the strongly magnetized, real plasma in thermal equilibrium approximated by the two dimensional, neutral quantum Coulomb gas in the grand canonical ensemble is expected to have a phase transition at $T \approx T_{c}$ and should describe interesting phenomena such as pair condensation below $T_{c}$.

Acknowledgements. I am indebted to E. Lieb and E. Seiler for many fruitful discussions and useful hints during the course of this work and to E. Lieb and A. Wightman for their enthusiasm and encouragement.

\section{The Connection between Classical Statistical Mechanics and Euclidean Field Theory}

In this section we explain the basic connection between CSM and Euclidean field theory (EFT) used in this paper. See $[7,28]$ for extensive information about EFT. We show how estimates in EFT yield estimates in CSM and vice versa. See $[6,1]$ for earlier discussions of the CSM-EFT connections explored below.

\section{a) Basic Definitions and a CSM Estimate}

In the following $K$ is the universal symbol for a constant; its value may vary from estimate to estimate. The class of potentials $V(x, y)$ we consider in this section is characterized in

Definition 2.1. Let $S_{R}$ be a sphere in $\mathbb{R}^{s}$ of radius $R \leqq \infty$ centered at the origin. Let $V(x, y)$ be the kernel of some positive operator $V$ on $L^{2}\left(S_{R}, d^{s} x\right)$ and assume that $V(x, y)$ is continuous in $x$ and $y$ and that

$$
\sup _{x, y \in S_{R}}|V(x, y)| \leqq K .
$$

Specifically, let $s=2, R=\infty$ and think of $V(x, y)$ as being given by

$$
V(x, y)=V_{\kappa, m}(x-y)=\int d^{2} x^{\prime} d^{2} y^{\prime} h_{\kappa}\left(x-x^{\prime}\right) h_{\kappa}\left(y-y^{\prime}\right) C_{m}\left(x^{\prime}-y^{\prime}\right),
$$

where $C_{m}(x-y)$ is the kernel of $\left(-\Delta+m^{2}\right)^{-1}, \Delta$ is the two dimensional Laplacian, $m>0$, and $h_{\kappa}$ is a positive, continuous function (the charge distribution of a particle) with

$$
\begin{aligned}
& \operatorname{supp} h_{\kappa} \cong\left\{x|| x \mid \leqq \frac{1}{\kappa}\right\}, h_{\kappa}(x)=h_{\kappa}(-x), \\
& \int h_{\kappa}(x) d^{2} x=1 \text { and } h_{\kappa}(x) \rightarrow \delta(x), \text { as } \kappa \rightarrow \infty .
\end{aligned}
$$


Here $\delta$ is the two dimensional $\delta$-function, and the convergence in (2.3) is on $C\left(S_{R}\right)$, the space of continuous functions over $S_{R} ; R \leqq \infty$.

In Section 3 we study the potentials $V_{\kappa, m}$ and show that one can let tend $\kappa \rightarrow \infty$ (corresponding to replacing particles with charge distribution $h_{\kappa}$ by point particles). In Section 4 we investigate the case where $m=0$.

Definition 2.2. Let $X^{n}=\left(x_{1}, \ldots, x_{n}\right)$ and $Y^{n}=\left(y_{1}, \ldots, y_{n}\right)$ be points in $\mathbb{R}^{s n}$ (particle configurations). Let

$$
d X^{n}=\prod_{i=1}^{n} d^{s} x_{i}, \quad \text { etc. }
$$

We set

$$
\begin{aligned}
U_{\Lambda}\left(X^{n}, Y^{n}\right)= & \varepsilon^{2} \sum_{1 \leqq i<j \leqq n}\left\{V\left(x_{i}, x_{j}\right)+V\left(y_{i}, y_{j}\right)\right\} \\
& -\varepsilon^{2} \sum_{i, j=1}^{n} V\left(x_{i}, y_{j}\right) .
\end{aligned}
$$

Let $g$ be some positive function in $L^{1}\left(S_{R}, d^{s} x\right)$ and

$$
g\left(X^{n}\right)=\prod_{i=1}^{n} g\left(x_{i}\right)
$$

We set

$$
\varrho_{n}(x)=\sum_{j=1}^{n} \varepsilon\left\{\delta\left(x-x_{j}\right)-\delta\left(x-y_{j}\right)\right\} .
$$

In the following we may set $\beta \equiv \frac{1}{k T}=1$ which can always be achieved by redefinition of the charge $\varepsilon$.

The classical, canonical partition function for $n$ positively and $n$ negatively charged particles with two body potential $V$ in an external potential $\log g\left(X^{n}\right)+$ $\log g\left(Y^{n}\right)$ is defined by

$$
\begin{aligned}
Z_{n}(V, g) & \equiv \int d X^{n} d Y^{n} g\left(X^{n}\right) g\left(Y^{n}\right) e^{-U_{V}\left(X^{n}, Y^{n}\right)} \\
& =\int d X^{n} d Y^{n} g\left(X^{n}\right) g\left(Y^{n}\right) e^{-\int d x d y \varrho_{n}(x) V(x, y) \varrho_{n}(y)} e^{\varepsilon^{2} \sum_{i=1}^{n}\left\{V\left(x_{i}, x_{i}\right)+V\left(y_{i}, y_{t}\right)\right\}} .
\end{aligned}
$$

Conventionally $g(x)=\chi_{\Lambda}(x)$, where $\chi_{\Lambda}$ is the characteristic function of some (open) region $\Lambda \subset \mathbb{R}^{s}$ with volume $|\Lambda|$. (We must be a little more general here; see $[8,9]$ for the reasons.)

Lemma 2.1. Suppose that for arbitrary, signed measures $\varrho$ with $\int d x \varrho(x)=0$, i.e. total charge 0 , supp $\varrho \subseteq S_{R}$,

$$
\int d x d y \varrho(x) V(x, y) \varrho(y) \geqq \int d x d y \varrho(x) W(x, y) \varrho(y)
$$

and that $\sup _{x \in S_{R}}\{V(x, x)-W(x, x)\} \leqq K$. 
Then

$Z_{n}(V, g) \leqq e^{2 n K} Z_{n}(W, g)$.

Proof.

$$
\begin{aligned}
Z_{n}(V, g)= & \int d X^{n} d Y^{n} g\left(X^{n}\right) g\left(Y^{n}\right) e^{-U_{W}\left(X^{n}, Y^{n}\right)} \\
& \cdot e^{-\int d x d y \varrho_{n}(x)(V-W)(x, y) \varrho_{n}(y)} \\
& \cdot e^{\sum_{i=1}^{n}\left\{V\left(x_{i}, x_{\imath}\right)-W\left(x_{\imath}, x_{\imath}\right)+V\left(y_{t}, y_{i}\right)-W\left(y_{l}, y_{l}\right)\right\}} \\
\leqq & e^{2 n K} \int d X^{n} d Y^{n} g\left(X^{n}\right) g\left(Y^{n}\right) e^{-U_{W}\left(X^{n}, Y^{n}\right)},
\end{aligned}
$$

since $\sup _{x \in S_{R}}\{V(x, x)-W(x, x)\} \leqq K$ and by (2.8). Q.E.D.

Remark. Since the signed measure $\varrho_{n}\left[\right.$ see (2.6)] satisfies $\int d x \varrho_{n}(x)=0$, i.e. $\tilde{\varrho}_{n}(0)=0$, Lemma 2.1, (2.8) hold for certain non-positive operators $V$. That is why Lemma 2.1 permits us to bound the Coulomb - from above by the Yukawa partition function, although the two-dimensional Coulomb potential is not the kernel of a positive operator; see Section 4. (I owe this remark to Seiler.)

Definition 2.3. The grand canonical partition function $Z(V, z g)$ for neutral systems is defined by

$$
Z(V, z g)=\sum_{n=0}^{\infty} \frac{z^{2 n}}{(n !)^{2}} Z_{n}(V, g)
$$

The r.h.s. of (2.11) is known to converge absolutely for all $z$ if the potential $V$ satisfies Definition 2.1 and $g$ is in $L^{1}\left(S_{R}, d^{s} x\right)$. This is essentially the same estimate as (2.10). For details see e.g. [26]. In subsection (c) we prove an estimate going in the opposite direction of the one proved in Lemma 2.1:

For

$$
V \geqq W \geqq 0 \quad Z(W, g) \leqq Z(V, 2 g) .
$$

\section{b) The EFT Formalism}

Without loss of generality we may assume that the null space of the operator

$V$ is $\{\mathbf{0}\}$.

(If not it can always be factored out.)

Let

$$
\mathscr{S}= \begin{cases}C_{0, \text { real }}^{\infty}\left(S_{R}\right) & \text { for } \quad R<\infty, \quad \text { and } \\ \mathscr{S}_{\text {real }}\left(\mathbb{R}^{s}\right) & \text { for } \quad R=\infty,\end{cases}
$$

where $\mathscr{S}_{\text {real }}\left(\mathbb{R}^{s}\right)$ is the real Schwartz space over $\mathbb{R}^{s}$. Let $\mathscr{S}^{\prime}$ denote the (topological) dual of $\mathscr{S}$. Points in $\mathscr{S}$ are denoted by $f, g, h \ldots$, points in $\mathscr{S}^{\prime}$ by $\Phi$.

Let $\Sigma$ be the $\sigma$-algebra on $\mathscr{S}^{\prime}$ generated by all Borel cylinder sets; see e.g. [14].

We define a functional $J_{V}$ on $\mathscr{S}$ :

$$
J_{V}(f)=e^{-\frac{1}{2}(f, V f)} .
$$


The functional $J$ is normalized, i.e. $J_{V}(0)=1$, continuous on $\mathscr{S}$ and of positive type $[14,22]$. By Minlos' theorem [22] it is the Fourier transform of some probability measure $d \mu_{V}$ on $\left(\mathscr{S}^{\prime}, \Sigma\right)$, i.e.

$$
J_{V}(f)=\int_{\mathscr{S}^{\prime}} d \mu_{V}(\Phi) e^{i \Phi(f)} .
$$

The measure $d \mu_{V}$ is the Gaussian measure on $\left(\mathscr{S}^{\prime}, \Sigma\right)$ with mean 0 and covariance operator $V$.

If $F$ is a $d \mu_{V}$-integrable function on $\mathscr{S}^{\prime}$ we set

$$
\langle\mathrm{F}\rangle_{V} \equiv \int_{\mathscr{S}^{\prime}} \mathrm{d} \mu_{V}(\Phi) \mathrm{F}(\Phi)
$$

Wick (normal) ordering of exponentials is defined by

$$
: e^{i \Phi(f)}:_{V} \equiv e^{i \Phi(f)}\left\langle e^{i \Phi(f)}\right\rangle_{V}^{-1}=e^{i \Phi(f)} e^{\frac{1}{2}(f, V f)} .
$$

Lemma 2.2. For $f_{1}, \ldots, f_{n}$ in $\mathscr{S}$

$$
\left\langle\prod_{j=1}^{n}: e^{i \Phi\left(f_{j}\right)}:_{V}\right\rangle_{V}=\exp \left[-\sum_{1 \leqq i<j \leqq n}\left(f_{i}, V f_{j}\right)\right] \geqq 0 \text {. }
$$

If the sequence $\left\{f_{j}^{l}(x)\right\}_{l=1}^{\infty}$ converges to $\varepsilon_{j} \delta\left(x-x_{j}\right)$ on $C\left(S_{R}\right)$, as $l \rightarrow \infty$, for all $j=1, \ldots, n$ then

$$
\lim _{l \rightarrow \infty}\left\langle\prod_{j=1}^{n}: e^{i \Phi\left(f_{J}^{l}\right)}:_{V}\right\rangle_{V} \equiv\left\langle\prod_{j=1}^{n}: e^{i \varepsilon, \Phi\left(x_{J}\right)}:_{V}\right\rangle_{V}=\exp \left[-\sum_{1 \leqq i<j \leqq n} \varepsilon_{i} \varepsilon_{j} V\left(x_{i}, x_{j}\right)\right] \geqq 0 .
$$

Proof. The first part is Wick's theorem and follows directly from (2.16) and (2.14); (2.17) follows from Definition 2.1, (boundedness and continuity of $V(x, y)$ ), and the first part of the lemma. See also [1]. Q.E.D.

Definition 2.4.

$$
\begin{aligned}
& \chi_{V}^{ \pm}(g) \equiv \int d^{s} x g(x): e^{ \pm i \varepsilon \Phi}:_{V}(x) \\
& : \cos \varepsilon \Phi:_{V}(g) \equiv \frac{1}{2}\left\{\chi_{V}^{+}(g)+\chi_{V}^{-}(g)\right\} .
\end{aligned}
$$

By (2.7) and Lemma 2.2

$$
Z_{n}(V, g)=\left\langle\left(\chi_{V}^{+}(g)\right)^{n}\left(\chi_{V}^{-}(g)\right)^{n}\right\rangle_{V} .
$$

For total charge $q$ we define

$$
Z_{2 n+q}^{q}(V, g)=\left\langle\left(\chi_{V}^{+}(g)\right)^{n+q}\left(\chi_{V}^{-}(g)\right)^{n}\right\rangle_{V} .
$$

Obviously

$$
\begin{aligned}
0 & \leqq Z_{2 n}^{2 q}(V, g) \leqq\left\langle\left|\chi_{V}^{+}(g)\right|^{n+q}\left|\chi_{V}^{-}(g)\right|^{n-q}\right\rangle_{V} \\
& =\left\langle\left(\chi_{V}^{+}(g)\right)^{n}\left(\chi_{V}^{-}(g)\right)^{n}\right\rangle_{V}=Z_{n}(V, g) .
\end{aligned}
$$

Using Definition 2.4 we see that

$$
\begin{aligned}
\frac{1}{n !}\left\langle: \cos \varepsilon \Phi:_{V}(g)^{n}\right\rangle_{V} & =2^{-n} \sum_{q=0}^{n} \frac{1}{q !(n-q) !}\left\langle\left(\chi_{V}^{+}(g)\right)^{q}\left(\chi_{V}^{-}(g)\right)^{n-q}\right\rangle_{V} \\
& =2^{-n} \sum_{q=0}^{n} \frac{1}{q !(n-q) !} Z_{n}^{2 q-n}(V, g) .
\end{aligned}
$$


By (2.20) and (2.21)

$$
\frac{1}{(2 n) !}\left\langle: \cos \varepsilon \Phi:_{V}(g)^{2 n}\right\rangle \leqq \frac{1}{(2 n) !} Z_{n}(V, g)<\frac{1}{(n !)^{2}} Z_{n}(V, g) \text {. }
$$

On the other hand all terms on the r.h.s. of (2.21) are positive by Lemma 2.2. Hence

$$
\frac{1}{(n !)^{2}} Z_{n}(V, g) \leqq \frac{2^{2 n}}{(2 n) !}\left\langle: \cos \varepsilon \Phi:_{V}(g)^{2 n}\right\rangle_{V} .
$$

Definition 2.4 and (2.21) lead us to the following

Definition 2.5. The grand canonical partition function of a system in a heat and charged particle - reservoir is defined by

$$
\begin{aligned}
\Xi(V, z g) & =\sum_{n=0}^{\infty} \frac{z^{n}}{n !}\left\langle: \cos \varepsilon \Phi:_{V}(g)^{n}\right\rangle_{V} \\
& \equiv\left\langle\exp \left\{: \cos \varepsilon \Phi:_{V}(z g)\right\}\right\rangle_{V} ;
\end{aligned}
$$

( $z$ is the fugacity). Clearly

$$
\begin{aligned}
|\Xi(V, z g)| & \leqq \Xi(V,|z| g) \leqq 2 \Xi_{\mathrm{cosh}}(V,|z| g) \\
& \equiv 2 \sum_{n=0}^{\infty} \frac{|z|^{2 n}}{(2 n) !}\left\langle: \cos \varepsilon \Phi:_{V}(g)^{2 n}\right\rangle_{V}
\end{aligned}
$$

and the convergence of the series on the r.h.s. of (2.25) for arbitrary $|z|$ follows from (2.22) and (2.11).

Equations and inequalities (2.18)-(2.25) yield

\section{Theorem 2.3.}
(1) $Z_{2 n}^{2 q}(V, g) \leqq Z_{n}(V, g)$
(2) $Z(V, g) \leqq \Xi_{\cosh }(V, 2 g)$
(3) $\Xi_{\cosh }(V, g) \leqq Z(V, g)$
(4) For positive $g$

$$
\Xi(V, g) \leqq 2 \Xi_{\mathrm{cosh}}(V, g) \leqq 2 \Xi(V, g) \text {. }
$$

Remark. The partition function $\Xi(V, g)$ plays an important role in the construction of the field theories described in the introduction under $B, \tilde{B}, C$ and $\tilde{C}$ if we set $V(x, y)=C_{m}(x-y)$; see $[8,9]$.

\section{c) An EFT Estimate (Conditioning)}

The following is a special case of a result due to Guerra, Rosen and Simon [16] called "conditioning".

Theorem 2.4. Let $V \geqq W \geqq 0$. Then for real-valued $g$

(1) $\Xi(V, \pm g) \geqq \Xi(W, \pm g)$,

(2) $Z(V, g) \geqq \Xi_{\cosh }(V, g) \geqq \Xi_{\cosh }(W, g) \geqq Z\left(W, \frac{1}{2} g\right)$,

(3) $\sum_{q=-n}^{n} \frac{1}{(n+q) !(n-q) !} Z_{2 n}^{2 q}(V, \pm g) \geqq \sum_{q=-n}^{n} \frac{1}{(n+q) !(n-q) !} Z_{2 n}^{2 q}(W, \pm g)$.

Proof. Clearly (2) follows from (1) by Theorem 2.3, (2) and (3). 
The idea of the proof of (1) and (3) is: Notice that

$$
\begin{aligned}
\Xi(V, g) & =\left\langle\exp \left\{: \cos \varepsilon \Phi:_{V}(g)\right\}\right\rangle_{V} \\
& =\left\langle\exp \left\{: \cos \varepsilon\left(\Phi_{1}+\Phi_{2}\right):_{V-W, W}(g)\right\}\right\rangle_{V-W, W},
\end{aligned}
$$

and

$$
\sum_{q=-n}^{n} \frac{1}{(n+q) !(n-q) !} Z_{2 n}^{2 q}(V, g)=\left\langle\left\{: \cos \varepsilon\left(\Phi_{1}+\Phi_{2}\right):_{V-W, W}(g)\right\}^{2 n}\right\rangle_{V-W, W}
$$

where $\Phi_{1}$ and $\Phi_{2}$ are two independent Gaussian random fields with covariance operator $V-W, W$, respectively. Then $\Phi_{1}+\Phi_{2}$ is a Gaussian random field with covariance $V$. Jensen's inequality gives:

$$
\begin{aligned}
& \left\langle\exp \left\{: \cos \varepsilon\left(\Phi_{1}+\Phi_{2}\right):_{V-W, W}(\mathrm{~g})\right\}\right\rangle_{V-W, W} \\
& \geqq\left\langle\exp \left\{\int_{\mathscr{S}^{\prime}} d \mu_{V-W}\left(\Phi_{1}\right): \cos \varepsilon\left(\Phi_{1}+\Phi_{2}\right):_{V-W, W}(g)\right\}\right\rangle \\
& =\left\langle\exp : \cos \varepsilon \Phi_{2}:_{W}(g)\right\rangle_{W} \quad \text { which proves }(1) .
\end{aligned}
$$

Moreover (3) follows by applying the Hölder inequality with respect to $\int_{\mathscr{S}^{\prime}} d \mu\left(\Phi_{1}\right)$ to the r.h.s. of $\left(2.26^{\prime}\right)$. For details see [16, 28]. Q.E.D.

Remark. Notice that Theorem 2.4, (2) and (3) goes in the opposite direction of Lemma 2.1, (2.9) which yields

$$
Z(V, g) \leqq Z\left(W, e^{K} g\right)
$$

where $K=\sup _{s \in S_{R}}\{V(x, x)-W(x, x)\}$.

\section{d) Decoupling of Nearest Neighbour Cubes}

Let $R=\infty$, i.e. $S_{R}=\mathbb{R}^{s}$. If $B$ is some region in $\mathbb{R}^{s} \chi_{B}$ denotes the characteristic function of $B$.

We now cover $\mathbb{R}^{s}$ with cubes $\Delta_{\underline{n}}$ of unit size centered at the points

$$
\underline{n}=\left(n^{1}, \ldots, n^{s}\right) \in \mathbb{Z}^{s}
$$

with faces parallel to the planes $x^{j}=0, j=1, \ldots, s$.

We then decompose each cube $\Delta_{\underline{n}}$ into $2^{s}$ disjoint cubes $\Delta_{\underline{n}}^{1}, \ldots, \Delta_{\underline{n}}^{2 s}$, each of volume $\left|\Delta_{\underline{n}}^{j}\right|=2^{-s}$.

Clearly

$$
: \cos \varepsilon \Phi:_{V}(g)=\sum_{j=1}^{2^{s}} \sum_{\underline{n} \in \mathbb{Z}^{s}}: \cos \varepsilon \Phi:_{V}\left(g \chi_{\Delta_{\underline{n}}^{J}}\right) .
$$

By Hölder's inequality

$$
\begin{aligned}
\Xi(V, g) & =\left\langle\exp \left\{: \cos \varepsilon \Phi:_{V}(g)\right\}\right\rangle_{V} \\
& \leqq \prod_{j=1}^{2^{s}}\left\langle\exp \left\{\sum_{\underline{n} \in \mathbb{Z}^{s}}: \cos \varepsilon \Phi:_{V}\left(2^{s} g \chi_{\Delta_{\underline{n}}^{j}}\right)\right\}\right\rangle_{V}^{2-s} \\
& =\prod_{j=1}^{2^{s}} \Xi\left(V, \sum_{\underline{n} \in \mathbb{Z}^{s}} 2^{s} g \chi_{\Delta_{\underline{n}}^{j}}\right)^{2-s} .
\end{aligned}
$$


By Theorem 2.3

$$
\begin{aligned}
Z(V, g) & \leqq \Xi_{\cosh }(V, 2 g) \leqq \Xi(V, 2 g) \\
& \leqq \prod_{j=1}^{2^{s}} \Xi\left(V, \sum_{\underline{n} \in \mathbb{Z}^{s}} 2^{s+1} g \chi_{\Delta_{\underline{n}}^{j}}\right)^{2-s} \\
& \leqq 2 \prod_{j=1}^{2^{s}} Z\left(V, \sum_{\underline{n} \in \mathbb{Z}^{s}} 2^{s+1} g \chi_{\Delta_{\underline{n}}^{j}}\right)^{2-s} .
\end{aligned}
$$

These simple inequalities decouple nearest neighbour cubes. We apply them in subsections e) and f) to prove stability.

\section{e) Strongly Decoupling Gaussian Measures}

Let $B$ be some cube in $\mathbb{R}^{s}$ and let $\Sigma_{B}$ denote the minimal $\sigma$-algebra on $\mathscr{S}^{\prime}$ such that all functions spanned by $\left\{e^{i \Phi(f)} \mid f \in \mathscr{S}\right.$, supp $\left.f \cong B\right\}$ are $\Sigma_{B}$-measurable.

Definition 2.6. The measure $d \mu_{V}$ is called a strongly decoupling Gaussian measure if there exists $p=p(V) \in[1, \infty)$ such that for arbitrary $\Sigma_{\Delta_{n}^{j}}$-measurable functions $F_{\underline{n}, j}, \underline{n} \in \mathbb{Z}^{s}$

$$
\left|\left\langle\prod_{\underline{n} \in \mathbb{Z}^{s}} F_{\underline{n}, j}\right\rangle_{V}\right| \leqq \prod_{\underline{n} \in \mathbb{Z}^{s}}\left\langle\left|F_{\underline{n}, j}\right|^{p}\right\rangle_{V}^{\frac{1}{p}},
$$

for arbitrary $j=1, \ldots, 2^{s}$.

If a potential $V \geqq 0$ satisfying Definition 2.1 has the property that the Gaussian measure $d \mu_{V}$ is strongly decoupling then using (2.28) and (2.30)

$$
\begin{aligned}
& \Xi(V, g) \leqq \prod_{j=1}^{2^{s}}\left\langle\prod_{\underline{n} \in \mathbb{Z}^{s}} \exp \left\{: \cos \varepsilon \Phi:_{V}\left(2^{s} g \chi_{\left.\Delta_{\underline{n}}\right)}\right)\right\}\right\}_{V}^{2^{-s}} \\
& \leqq \prod_{j=1}^{2^{s}} \prod_{\underline{n} \in \mathbb{Z}^{s}} \Xi\left(V, 2^{s} p g \chi_{\Delta_{\underline{n}}^{j}}\right)^{2^{-s} p^{-1}},
\end{aligned}
$$

and we have used the obvious fact that $\exp \left\{: \cos \varepsilon \Phi:_{V}\left(2^{s} g \chi_{\Lambda_{n}^{\prime}}\right)\right\}$ is a $d \mu_{V}$-integrable $\Sigma_{\Delta_{n}^{y}}$-measurable function on $\mathscr{S}^{\prime}$, for $g \in L^{1}\left(S_{R}, d^{s} x\right)$ which follows from (2.24), (2.25), (2.11).

By Theorem 2.3 and (2.29)

$$
Z(V, g) \leqq \prod_{j=1}^{2 s} \prod_{\underline{n} \in \mathbb{Z}^{s}}\left(2 Z\left(V, 2^{s+1} p g \chi_{\underline{n}_{\underline{n}}^{j}}\right)^{2^{-s} p^{-1}} .\right.
$$

If $V$ is translation invariant $(R=\infty), g=\lambda \cdot \chi_{A}$, for some open region $\Lambda \subset \mathbb{R}^{s}$, $|\Lambda|$ the total volume of the cubes $\Delta_{n}$ intersecting $\Lambda$, and

$$
Z\left(V, \lambda \chi_{\Delta_{0}^{1}}\right) \leqq K_{\lambda}<\infty,
$$

for arbitrary, positive $\lambda$ then with $\bar{K}_{\lambda}=\frac{1}{2^{s} p} \log 2 K_{2^{s} p \lambda}$

$$
\Xi\left(V, \lambda \chi_{A}\right) \leqq e^{\bar{K}_{\lambda}|\Lambda|} .
$$

For complex $z$ we have

$$
|\Xi(V, z g)| \leqq \Xi(V, \operatorname{Re} z g),
$$


thus

$$
\left|\Xi\left(V, z \chi_{A}\right)\right| \leqq e^{\bar{K}_{\mathrm{Re} z}|\Lambda|},
$$

and by (2.32)

$$
\left|Z\left(V, z \chi_{A}\right)\right| \leqq Z\left(V,|z| \chi_{A}\right) \leqq e^{\bar{K}_{2|z|}|\Lambda|} .
$$

We have proved

Theorem 2.5. If $V$ is translation invariant and $d \mu_{V}$ is strongly decoupling the finite volume estimate (2.33) implies the stability estimates (2.34), (2.35).

\section{f) Application to the Yukawa Potential}

Let

$$
V(x, y)=V_{\kappa, m}(x-y)=\int d^{s} x^{\prime} d^{s} y^{\prime} h_{k}\left(x^{\prime}-x\right) C_{m}\left(x^{\prime}-y^{\prime}\right) h_{\kappa}\left(y^{\prime}-y\right),
$$

where $C_{m}(x-y)$ is the kernel of $\left(-\Delta+m^{2}\right)^{-1}$.

Let $\Phi_{\kappa} \equiv h_{\kappa} * \Phi$. The following equation is useful:

$$
\langle F(\Phi)\rangle_{V_{\kappa, m}}=\left\langle F\left(\Phi_{\kappa}\right)\right\rangle_{C_{m}} .
$$

To prove (2.37) we must only show that

$$
J_{V_{\kappa, m}}(f) \equiv\left\langle e^{i \Phi(f)}\right\rangle_{V_{\kappa, m}}=\left\langle e^{i \Phi_{\kappa}(f)}\right\rangle_{C_{m}}=J_{C_{m}}\left(h_{\kappa} * f\right)
$$

which follows directly from (2.36) and (2.14).

Theorem 2.6. Let $m$ be positive and $\kappa \geqq 4$, i.e. $\operatorname{supp} h_{\kappa} \leqq\left\{x|| x \mid \leqq \frac{1}{4}\right\}$. Then $d \mu_{V_{\kappa, m}}$ is a strongly decoupling Gaussian measure with $p=p\left(V_{\kappa, m}\right) \leqq p(m) \in(2, \infty)$, where $p(m)$ is independent of $\kappa$. Theorem 2.5 applies.

Proof. Nelson has shown that $d \mu_{C_{m}}$ has the Markov property [23], and is hypercontractive $[23,24] ;\left(d \mu_{C_{m}}\right.$ is Markovian, since the inverse of $C_{m}$, i.e. $-\Delta+m^{2}$, is a local operator, see [23], and hypercontractive, since $m>0$; see $[23,24,28]$ ). Let $\overline{\Delta_{n}^{j}}$ be the set of points $x \in \mathbb{R}^{s}$ with distance less than or equal to $\frac{1}{4}$ from $\Delta_{\underline{n}}^{j}$. For fixed $j$ the sets $\overline{\Delta_{\underline{n}}^{j}}, \overline{\Delta_{\underline{n}}^{j}}$ are non-overlapping for $\underline{n} \neq \underline{n}^{\prime}$. Applying Eq. (2.37) we observe that we must prove the decoupling estimate (2.30) for

$$
\left|\left\langle\prod_{\underline{n} \in \mathbb{Z}^{s}} \exp \left\{: \cos \varepsilon \Phi_{\kappa}:_{C_{m}}\left(z g \chi \Delta_{\underline{n}}^{j}\right)\right\}\right\rangle_{C_{m}}\right| \text {. }
$$

Obviously $\exp \left\{: \cos \varepsilon \Phi_{\kappa}:_{C_{m}}\left(z g \chi_{\Delta_{\underline{n}}^{J}}\right)\right\}$ is $\Sigma_{\overline{\Delta_{n}^{j}}}$-measurable. Therefore the Markov property and hypercontractivity for $d \mu_{C_{m}}$ imply the decoupling estimate (2.30) for (2.38). The simple proof of

$$
\left.\begin{array}{l}
\text { Markov property }+ \\
\text { hypercontractivity }
\end{array}\right\} \Rightarrow(2.38) \text { satisfies (2.30) }
$$

is due to Guerra, Rosen and Simon and is called the "checker board" estimate. For details see $[16,28]$. Q.E.D.

Remarks. Theorems 2.6 and 2.5 show that we have proved stability for CSM with Yukawa forces in two dimensions once we have proved stability in a finite 
volume, i.e. inequality (2.31). It then follows from Lemma 2.1 and (2.27) by a simple argument (see Section 4) that the neutral Coulomb CSM system in two dimensions is stable, too. These results are basic for the construction of the field theories $B, \tilde{B}, C$ and $\tilde{C}$, since there is indeed strictly no difference between CSM $A, \tilde{A}$ in the grand canonical ensemble and the field theories $B, \tilde{B}$, respectively, in the Euclidean formulation; see $[8,9]$.

\section{The Heart of the Proof: Stability in a Finite Volume}

In this section we show that for $\beta \varepsilon^{2}<4 \pi$ (or $\varepsilon^{2}<4 \pi$ if we set $\beta=1$ ) the CSM system with Yukawa forces is stable. The Yukawa potential is given by

$$
V(x, y)=C_{m}(x-y) \text {, and } m>0 \text {. }
$$

It is convenient to first consider CSM for the regularized Yukawa potential $V_{\kappa, m}-$ see (2.36) - where the cutoff function $h_{\kappa}$ satisfies Definition 2.1, (2.3), and to derive estimates which are uniform in $\kappa$. By the results of Section 2, e) and f), we are left with proving that $Z\left(V_{\kappa, m}, \lambda \chi_{\Delta}\right)$ is uniformly bounded in $\kappa$, for arbitrary, fixed, positive $\lambda$ and $\Delta$ a square of volume $1 / 4$.

The strategy of the proof is to reduce this problem to the one solved by Deutsch and Lavaud in [4] for the Coulomb system, i.e. to bound the partition function with Yukawa forces from above by the one with Coulomb-like forces which can be estimated explicitly. This is possible by using conditioning (Theorem 2.4). Of course we have to replace the Coulomb potential by one which is the kernel of a positive operator. It is convenient to work with the grand canonical partition function $\Xi\left(V_{\kappa, m}, g\right)$.

\section{a) Reduction to Coulomb-like Potentials}

Let $\Delta_{\frac{1}{2}}, \Delta_{1}$ be the squares of volume $1 / 4,1$, respectively, with boundaries parallel to the coordinate axes and centered at the point $(1,0)$. Let $S=S_{2}$ be the circular disk of radius 2 centered at the origin. Let $\Delta_{S}$ be the Laplacian on $L^{2}\left(S, d^{2} x\right)$ with 0 -Dirichlet data at $\partial S$. Finally let $C_{m}^{S}(x, y)$ be the kernel of $\left(-\Delta_{S}+m^{2}\right)^{-1}$. Notice that because of 0 -Dirichlet data at $\partial S-\Delta_{S}$ is strictly positive and hence $\left(-\Delta_{S}\right)^{-1}$ is a bounded, positive operator with kernel $C_{0}^{S}(x, y): C_{0}^{S} \in \bigcap_{p<\infty} L^{p}\left(S \times S, d^{2} x \times d^{2} x\right)$.
For all $x$ and $y$ in $\Delta_{1}$ and $m>0$

$$
0 \leqq\left[C_{m}-C_{m}^{S}\right](x, y) \leqq K .
$$

Lemma 3.1. Let $F$ be a $\Sigma_{\Delta_{1}}$-measurable function on $\mathscr{S}^{\prime}$. Then

$$
\left|\langle F\rangle_{C_{m}}\right| \leqq\left\langle|F|^{r}\right\rangle_{C_{m}^{s}}^{1 / r}
$$

for some $r=r(m, d)$ which is finite for $m>0$ and $d>0$, and $d$ is the distance between $\Delta_{1}$ and $\partial S$.

This lemma is proven in [16]. See also [28, Theorems I.23, VII.2]. For a general study of Gaussian measures on $\mathscr{S}^{\prime}$ see $[28,10]$. We set

$$
\begin{aligned}
V_{\kappa, m}^{S}(x, y) & =\int d^{2} x^{\prime} d^{2} y^{\prime} h_{\kappa}\left(x-x^{\prime}\right) h_{\kappa}\left(y-y^{\prime}\right) C_{m}\left(x^{\prime}, y^{\prime}\right) \\
& \equiv\left[\left(h_{\kappa} \otimes h_{\kappa}\right) * C_{m}^{S}\right](x, y),
\end{aligned}
$$


and

$$
\left.\begin{array}{rl}
w_{\kappa}(x) & =e^{-\frac{1}{2}\left[V_{\kappa, m}-V_{\kappa, m}^{S}\right](x, x)} \cdot \chi_{\Delta_{1}}(x), \\
w(x) & =e^{-\frac{1}{2}\left[C_{m}-C_{m}^{S}\right](x, x)} \cdot \chi_{\Delta_{1}}(x) . \\
\text { Notice that } w_{\kappa} \rightarrow w, \text { as } \kappa \rightarrow \infty, \text { in the }\|\cdot\|_{\infty} \text {-norm }
\end{array}\right\}
$$

Let $g$ be a positive function in $L^{1}\left(\Delta_{\frac{1}{2}}, d^{2} x\right)$. Then Lemma 3.1 yields

\section{Corollary 3.2.}

$$
\begin{aligned}
\Xi\left(V_{\kappa, m}, g\right) & =\left\langle\exp \left\{: \cos \varepsilon \Phi_{\kappa}:_{C_{m}}(g)\right\}\right\rangle_{C_{m}} \\
& \leqq\left\langle\exp \left\{: \cos \varepsilon \Phi_{\kappa}:_{C_{m}^{S}}\left(r \cdot w_{\kappa} \cdot g\right)\right\}\right\rangle_{C_{m}^{S}}^{1 / r} \\
& =\Xi\left(V_{\kappa, m}^{S}, r \cdot w_{\kappa} \cdot g\right)^{1 / r} \\
& \leqq \Xi\left(V_{\kappa, 0}^{S}, r \cdot w_{\kappa} \cdot g\right)^{1 / r} \\
& \leqq\left(2 Z\left(V_{\kappa, 0}^{S}, r \cdot w_{\kappa} \cdot g\right)\right)^{1 / r} .
\end{aligned}
$$

Proof. The first equation follows from (2.37). By the definition of Wick ordering (2.16) and (3.2)

$$
: e^{ \pm i \varepsilon \Phi_{\kappa}}:_{C_{m}}(g)=: e^{ \pm i \varepsilon \Phi_{\kappa}}:_{C_{m}^{S}}\left(w_{\kappa} \cdot g\right),
$$

for supp $g \subseteq \Delta_{\frac{1}{2}}$, whence

$$
\exp \left\{: \cos \varepsilon \Phi_{\kappa}:_{C_{m}}(g)\right\}=\exp \left\{: \cos \varepsilon \Phi_{\kappa}:_{C_{m}^{S}}\left(w_{\kappa} g\right)\right\} .
$$

This equation and Lemma 3.1 prove (3.3); (3.4) follows from the definition of $\Xi$; (3.5) follows from the inequality $\left(-\Delta_{S}\right)^{-1} \geqq\left(-\Delta_{S}+m^{2}\right)^{-1} \geqq 0$ by the conditioning inequality: Theorem 2.4, (1). Finally (3.6) follows from Theorem 2.3, (3) and (4). Q.E.D.

We are now left with estimating $Z\left(V_{\kappa, 0}^{S}, r w_{\kappa} g\right)$, uniformly in $\kappa$ and (as a result) showing that

$$
Z\left(V_{\kappa, m}, g\right) \rightarrow Z\left(C_{m}, g\right), \quad \text { as } \quad \kappa \rightarrow \infty \text {, etc. }
$$

\section{b) An Explicit Representation of the Green's Function $C_{0}^{S}(x, y)$}

Let $x=\left(x^{1}, x^{2}\right)$ and $y=\left(y^{1}, y^{2}\right)$ be points in $\mathbb{R}^{2}$. We set $z=x^{1}+i x^{2}$ and $w=y^{1}+i y^{2}$. We let $\hat{z}$ be the reflection of $z$ at the circle $\partial S$, i.e. $\hat{z}=\frac{4}{\bar{z}}$. If $z \in \partial S$ then $z=\hat{z}$.

\section{Lemma 3.3.}

$$
\begin{aligned}
C_{0}^{S}(x, y) & \equiv C_{0}^{S}(z, w) \equiv G^{S}(z, w) \\
& =-\frac{1}{4 \pi}\{\ln |z-w|+\ln |\hat{z}-\hat{w}|-\ln |\hat{z}-w|-\ln |z-\hat{w}|\} .
\end{aligned}
$$

Proof. In order to show that $G^{S}(z, w)$ defined by (3.8) is the Green's function of $-\Delta_{S}$ we must show that $1^{\circ}$ ) for $z$ or $w$ on $\partial S G^{S}(z, w)=0$, and $2^{\circ}$ ) for $w \notin \partial S$ $-\Delta_{S, z} G(z, w)=\delta(z-w)$, where

$$
-\Delta_{S, z}=-\frac{\partial^{2}}{\left(\partial x^{1}\right)^{2}}-\frac{\partial^{2}}{\left(\partial x^{2}\right)^{2}}
$$


with 0-Dirichlet data at $\partial S$. Obviously for $z$ or $w$ on $\partial S$ the r.h.s. of (3.8) vanishes by (3.7), whence $1^{\circ}$ ).

$G^{S}(z, w)=-\frac{1}{2 \pi}\{\ln |z-w|-\ln |4-z \bar{w}|+\ln 4\}$, by a trivial computation. If $w \notin \partial S$ then $-\ln |4-z \bar{w}|+\ln 4$ is a harmonic function on $S ;-\frac{1}{2 \pi} \ln |z-w|$ is the two dimensional Coulomb potential, hence by Green's theorem

$$
\left.-\Delta_{S, z} G^{S}(z, w)=\frac{1}{2 \pi} \Delta_{S, z} \ln |z-w|=\delta(z-w), \quad \text { i.e. } 2^{\circ}\right) \text {. Q.E.D. }
$$

We are now ready to estimate $Z\left(V_{\kappa, 0}^{S}, r w_{\kappa} g\right)$. Let $f_{\kappa} \equiv r \cdot w_{\kappa} \cdot g, f_{\kappa}\left(X^{n}\right) \equiv \prod_{j=1}^{n} f_{\kappa}\left(x_{j}\right)$, and $h_{\kappa}^{\otimes n} \otimes h_{\kappa}^{\otimes n}\left(X^{n}, Y^{n}\right) \equiv \prod_{j=1}^{n} h_{\kappa}\left(x_{j}\right) h_{\kappa}\left(y_{j}\right)$. Then

$$
\begin{aligned}
& Z_{n}\left(V_{\kappa, 0}^{S}, r \cdot w_{\kappa} \cdot g\right) \\
& =\int d X^{n} d Y^{n} f_{\kappa}\left(X^{n}\right) f_{\kappa}\left(Y^{n}\right) e^{-U_{V_{\kappa}, 0}^{S}\left(X^{n}, Y^{n}\right)} \\
& =\int d X^{n} d Y^{n} f_{\kappa}\left(X^{n}\right) f_{\kappa}\left(Y^{n}\right) e^{-\left[\left(h_{\kappa}^{\otimes n} \otimes h_{\kappa}^{\otimes n}\right) * U_{C_{0}^{S}}\right]\left(X^{n}, Y^{n}\right)} \\
& \leqq \int d X^{n} d Y^{n}\left(h_{\kappa}^{\oplus^{n}} * f_{\kappa}\right)\left(X^{n}\right)\left(h_{\kappa}^{\oplus n} * f_{\kappa}\right)\left(Y^{n}\right) e^{-U_{\mathcal{C}_{0}^{S}}\left(X^{n}, Y^{n}\right)}
\end{aligned}
$$

by Jensen's inequality.

Definition 3.1.

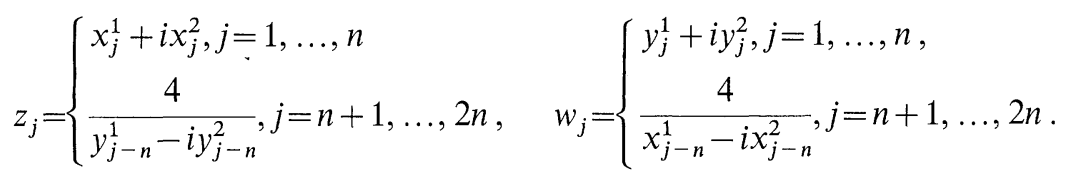

Notice that $\hat{z}_{j}=w_{j+n}$ and $\hat{w}_{j}=z_{j+n}$, for $j=1, \ldots, n$.

We set $Z^{n}=\left(z_{1}, \ldots, z_{n}\right)$ and $d Z^{n}=d X^{n}$,

$$
W^{n}=\left(w_{1}, \ldots, w_{n}\right) \text {, etc. }
$$

Finally we define $H_{\kappa}\left(g \mid Z^{n}, W^{n}\right)=\left(h_{\kappa}^{\otimes n} * f_{\kappa}\right)\left(Z^{n}\right)\left(h_{\kappa}^{\otimes n} * f_{\kappa}\right)\left(W^{n}\right)$ and observe that $H_{\kappa}\left(g \mid Z^{n}, W^{n}\right) \rightarrow(w g)\left(Z^{n}\right)(w g)\left(W^{n}\right), \quad$ as $\quad \kappa \rightarrow \infty$;

we let $\alpha \equiv \frac{\varepsilon^{2}}{4 \pi}\left(=\frac{\beta \varepsilon^{2}}{4 \pi}\right.$, for $\left.\beta=1\right)$. Then by Definition 2.2, (2.4) and (3.8)

$$
\begin{aligned}
e^{-U_{C_{0}^{S}}\left(Z^{n}, W^{n}\right)}= & \frac{\prod_{1 \leqq i<j \leqq 2 n}\left|z_{i}-z_{j}\right|^{\alpha}\left|w_{i}-w_{j}\right|^{\alpha}}{\prod_{i, j=1}^{2 n}\left|z_{i}-w_{j}\right|^{\alpha}} \\
& \times \prod_{j=1}^{n}\left|z_{j}-w_{j+n}\right|^{\alpha}\left|w_{j}-z_{j+n}\right|^{\alpha}
\end{aligned}
$$


and the second factor on the r.h.s. of (3.11) accounts for the fact that according to (3.8) a charged particle at $z_{j}$ does not interact with its image (with respect to $\partial S$ ) at $\hat{z}_{j}=w_{j+n}$.

By assumption supp $g \subseteq \Delta_{\frac{1}{2}}$. Hence [by Definition 2.1, (2.3) and the definition of $f_{\kappa}\left(Z^{n}\right)$ and $\left.H_{\kappa}\left(g \mid Z^{n}, W^{n}\right)\right]$

$$
\operatorname{supp} H_{\kappa}\left(g \mid Z^{n}, W^{n}\right) \subseteq \Delta_{1}^{\times 2 n}, \quad \text { for } \quad \kappa \geqq 4 \text {. }
$$

Notice that $\Delta_{1}$ does not contain the origin and that the maximal distance between points in $\Delta_{1}$ and in its image $\hat{\Delta}_{1}$ (with respect to $\partial S$ ) is therefore bounded. Hence

$$
\prod_{j=1}^{n}\left|z_{j}-w_{j+n}\right|^{\alpha}\left|w_{j}-z_{j+n}\right|^{\alpha} \leqq K_{1}^{n}
$$

on $\operatorname{supp} H_{\kappa}(g \mid \cdot)$, for some finite constant $K_{1}$.

\section{c) The Main Estimate}

By a lemma of Cauchy's - see [4] and refs. given there -

$$
\frac{\prod_{1 \leqq i<j \leqq 2 n}\left|z_{i}-z_{j}\right|^{\alpha}\left|w_{i}-w_{j}\right|^{\alpha}}{\prod_{i, j=1}^{2 n}\left|z_{i}-w_{j}\right|^{\alpha}}=\left|\operatorname{Det}\left[\frac{1}{z_{i}-w_{j}}\right]\right|^{\alpha}
$$

where $\left[\frac{1}{z_{i}-w_{j}}\right]$ denotes the $2 n \times 2 n$ matrix with matrix elements $\frac{1}{z_{i}-w_{j}}$.

Equations (3.13) and (3.11), (3.12) enable us to prove

Theorem 3.4. Let $\alpha<1\left(\right.$ i.e. $\left.\frac{\beta \varepsilon^{2}}{4 \pi}<1\right)$. Let $p \geqq 1$ be any real number such that $\alpha \cdot p<1$. Let $p^{\prime}=\frac{p}{p-1}$ and let $g$ be a function in $L^{p^{\prime}}\left(\Delta_{\frac{1}{2}}, d^{2} x\right)$. Then

$$
\left|Z_{n}\left(V_{\kappa, 0}^{S}, r w_{\kappa} g\right)\right| \leqq((2 n) !)^{\frac{1}{p}} K_{p}^{n}\|g\|_{p}^{2 n},
$$

uniformly in $\kappa \geqq 4$.

Proof. Let $H \in L^{p^{\prime}}\left(\Delta_{1}^{\times 2 n}, d Z^{n} d W^{n}\right)$. Then by (3.12) and Hölder's inequality

$$
\begin{aligned}
& \left|\int d Z^{n} d W^{n} H\left(Z^{n}, W^{n}\right)\right| \operatorname{Det}\left[\left.\frac{1}{z_{i}-w_{j}}\right|^{\alpha} \cdot \prod_{i=1}^{n}\left|z_{j}-w_{j+n}\right|^{\alpha}\left|w_{j}-z_{j+n}\right|^{\alpha} \mid\right. \\
& \leqq K_{1}^{n}\|H\|_{p^{\prime}}\left[\int_{\Delta_{1}^{\times 2 n}} d Z^{n} d W^{n}\left|\operatorname{Det}\left[\frac{1}{z_{i}-w_{j}}\right]\right|^{\alpha \cdot p}\right]^{1 / p} \\
& \leqq K_{1}^{n}\|H\|_{p^{\prime}}\left[\sum_{\pi \in \gamma_{2 n}} \int_{\Delta x_{1}^{\times 2 n}} d Z^{n} d W^{n} \prod_{j=1}^{2 n} \frac{1}{\left|z_{j}-w_{\pi(j)}\right|^{\alpha \cdot p}}\right]^{1 / p} .
\end{aligned}
$$

Since $\alpha p<1, \prod_{j=1}^{2 n} \frac{1}{\left|z_{j}-w_{\pi(j)}\right|^{\alpha \cdot p}}$ is $d Z^{n} d W^{n}-$ integrable on $\Delta_{1}^{\times 2 n}$, and

$$
\int_{\Delta_{1}^{\times 2 n}} d Z^{n} d W^{n} \prod_{j=1}^{2 n} \frac{1}{\left|z_{j}-w_{\pi(j)}\right|^{\alpha \cdot p}} \leqq K_{2}(p)^{n},
$$


for some constant $K_{2}(p)$ which is finite for $\alpha \cdot p<1$, independent of the permutation $\pi \in \gamma_{2 n}$.

Hence

$$
\begin{aligned}
& \left|\int d Z^{n} d W^{n} H\left(Z^{n}, W^{n}\right)\right| \operatorname{Det}\left[\frac{1}{z_{i}-w_{j}}\right]\left|\prod_{j=1}^{\alpha}\right| z_{j}-\left.w_{j+n}\right|^{\alpha}\left|w_{j}-z_{j+n}\right|^{\alpha} \mid \\
& \leqq\left(K_{1} K_{2}(p)\right)^{n}\|H\|_{p^{\prime}}((2 n) !)^{\frac{1}{p}} .
\end{aligned}
$$

For $H\left(Z^{n}, W^{n}\right)=r^{2 n}\left(h_{\kappa}^{\otimes n} * w_{\kappa} g\right)\left(Z^{n}\right)\left(h_{\kappa}^{\otimes n_{*}} w_{\kappa} g\right)\left(W^{n}\right)$ we get for $p^{\prime}<\infty$

$$
\begin{aligned}
\|H\|_{p^{\prime}} & \leqq r^{2 n} \| \tilde{h}_{\kappa}\left(w_{\kappa} g\right) \tilde{\sim}_{p}^{2 n} \\
& \leqq r^{2 n}\left\|\left(w_{\kappa} g\right) \tilde{\|}_{p}^{2 n} \leqq r^{2 n}\right\| w_{\kappa} g \|_{p^{\prime}}^{2 n} \\
& \leqq r^{2 n} K_{3}^{n}\|g\|_{p^{\prime}}^{2 n},
\end{aligned}
$$

by the Hausdorff-Young inequality and the facts that $\left|\tilde{h}_{\kappa}(k)\right| \leqq \int h_{\kappa}(x) d x=1$, and $\left\|w_{\kappa}\right\|_{\infty} \leqq \sqrt{K_{3}}$, for some finite constant $K_{3}$; see (3.1) and (3.2).

If we set $K_{p} \equiv r^{2} K_{1} K_{2}(p) K_{3}$, (3.14) now follows from (3.9), (3.15) and (3.16).

Combining Theorem 3.4 with Corollary 3.2 we get

Q.E.D.

Corollary 3.5. (Stability in the grand canonical ensemble.)

Let $\alpha<p \alpha<1, m>0$ and $\kappa \geqq 4$.

(1) Let $g \in L^{p^{\prime}}\left(\Delta, d^{2} x\right)$ with $p^{\prime}>\frac{1}{1-\alpha}$ (i.e. $\left.p \alpha<1\right)$, for some square $\Delta$ of finite volume. Then $\left\{\Xi\left(V_{\kappa, m}, z g\right)\right\}_{\kappa<\infty}$ and $\left\{Z\left(V_{\kappa, m}, z g\right)\right\}_{\kappa<\infty}$ are families of entire functions of $z$ which are bounded by

$$
K_{p} e^{\bar{K}_{p}|z| p / p-1}
$$

for some constants $K_{p}, \bar{K}_{p}$ which are finite for $\alpha<p \alpha<1$ and uniformly in $\kappa$.

(2) Let $\Lambda$ be an open set in $\mathbb{R}^{2}$ with the property that the total volume of a covering of $\Lambda$ by unit squares is bounded by some constant times $|\Lambda|$. Then

$$
\left|\Xi\left(V_{\kappa, m}, z \cdot \chi_{\Lambda}\right)\right| \leqq K_{p} e^{\vec{K}_{p}|\operatorname{Re} z|^{p / p-1}|\Lambda|},
$$

and

$$
\left|Z\left(V_{\kappa, m}, z \cdot \chi_{\Lambda}\right)\right| \leqq K_{p} e^{\bar{K}_{p}|z|^{p / p-1}|\Lambda|},
$$

for some constants $K_{p}, \bar{K}_{p}$ which are finite for $\alpha<p \cdot \alpha<1$, and uniformly in $\kappa$.

Proof. The first part is a direct consequence of Corollary 3.2 and inequality (3.14); (2) follows by combining (1) with Theorems 2.5 and 2.6.

Corollary 3.6. (Stability in the canonical ensemble.)

Let $\alpha<p \alpha<1, \kappa \geqq 4$ and set $\varrho \equiv \frac{n}{|\Lambda|}$. Then

$$
\left|Z_{n}\left(V_{\kappa, m}, z \cdot \chi_{\Lambda}\right)\right| \leqq K_{p}^{n}|z|^{2 n}\left(\varrho^{\frac{1-p}{p}}\right)^{2 n}(n !)^{2}
$$


and

$$
K\left\{: \cos \varepsilon \Phi_{\kappa}:_{C_{m}}\left(z \cdot \chi_{\Lambda}\right)\right\}^{n}>\left._{C_{m}}\left|\leqq K_{p}^{n}\right| z\right|^{n}\left(\varrho^{\frac{1-p}{p}}\right)^{n} n !,
$$

for all $\varrho>0$ and uniformly in $\kappa$. Here $K_{p}$ are constants which are finite for $\alpha<p \alpha<1$.

Proof. By Corollary $3.5 Z\left(V_{\kappa, m}, z \cdot \chi_{1}\right)$ is an entire function of $z$ the absolute value of which is bounded by $K_{p} e^{K_{p}|z|^{p / p-1}|\Lambda|}$. Choose as a contour $\Gamma$ in the $z$-plane the circle of radius $\varrho^{p-1 / p}$ centered at the origin and apply the Cauchy integral formula for $\Gamma$ to compute the $2 n^{\text {th }}$ derivative of $Z\left(V_{\kappa, m}, z \chi_{A}\right)$ at $z=0$; (3.17) now follows immediately; the same argument applied to $\Xi\left(V_{\kappa, m}, z \chi_{A}\right)$ yields (3.18).

Theorem 3.7. (Removing the cutoff $\kappa$.) Let $\alpha<p \alpha<1$ and $m>0$.

Q.E.D.

(1) Let $g \in L^{p^{\prime}}\left(\Delta, d^{2} x\right)$ for some $p^{\prime}>\frac{1}{1-\alpha}$ (i.e. $\left.p \alpha<1\right)$ and some square $\Delta$ of finite volume. Then

$$
\begin{aligned}
\lim _{\kappa \rightarrow \infty} Z_{n}\left(V_{\kappa, m}, g\right) & =Z_{n}\left(C_{m}, g\right) \\
\lim _{\kappa \rightarrow \infty}\left\langle\left\{: \cos \varepsilon \Phi_{\kappa}:_{C_{m}}(g)\right\}^{n}\right\rangle_{C_{m}} & =\left\langle\left\{: \cos \varepsilon \Phi:_{C_{m}}(g)\right\}^{n}\right\rangle_{C_{m}} \\
\lim _{\kappa \rightarrow \infty} \Xi\left(V_{\kappa, m}, z \cdot g\right) & =\Xi\left(C_{m}, z \cdot g\right), \text { etc. }
\end{aligned}
$$

(2) The bounds of Corollaries 3.5 and 3.6 hold for $\Xi\left(V_{\kappa, m}, \cdot\right)$ replaced by $\Xi\left(C_{m}, \cdot\right)$, $Z_{n}\left(V_{\kappa, m}, \cdot\right)$ replaced by $Z_{n}\left(C_{m}, \cdot\right)$, etc.

Proof. Under the hypotheses of Theorem 3.7

$$
g\left(X^{n}\right) g\left(Y^{n}\right) e^{-U_{C_{m}}\left(X^{n}, Y^{n}\right)} \text { and } e^{-p U_{C_{m}}\left(X^{n}, Y^{n}\right)}
$$

are $d X^{n} d Y^{n}$-integrable for all $p$ with $p \alpha<1$. (Notice that $C_{m}(x) \sim-\frac{1}{4 \pi} \ln \left(m^{2}|x|^{2}\right)$, as $x \rightarrow 0$.). See also [4]. We now show that

$$
g\left(X^{n}\right) g\left(Y^{n}\right) e^{-U_{V_{k}, m}\left(X n, Y^{n}\right)} \rightarrow g\left(X^{n}\right) g\left(Y^{n}\right) e^{-U_{\mathcal{C}_{m}}\left(X^{n}, Y^{n}\right)},
$$

as $\kappa \rightarrow \infty$, in $L^{1}\left(\Delta^{\times 2 n}, d X^{n} \cdot d Y^{n}\right)$.

By the Hölder inequality this follows from

$$
e^{-U_{V_{\kappa}, m}\left(X^{n}, Y^{n}\right)} \rightarrow e^{-U_{C_{m}}\left(X^{n}, Y^{n}\right)}, \text { as } \kappa \rightarrow \infty,
$$

in $L^{p}\left(\Delta^{\times 2 n}, d X^{n} d Y^{n}\right)$. Thus we must show that

$$
I_{\kappa} \equiv \int_{\Delta^{\times 2 n}} d X^{n} d Y^{n}\left|e^{-p U_{V_{\kappa}, m}\left(X^{n}, Y^{n}\right)}-e^{-p U_{\mathcal{C}_{m}}\left(X^{n}, Y^{n}\right)}\right| . \quad \rightarrow 0, \text { as } \kappa \rightarrow \infty .
$$

Now

$$
\begin{aligned}
& I_{\kappa} \leqq \int_{\Delta^{\times 2 n}} d X^{n} d Y^{n} \int_{0}^{1} d s e^{-\left(s p U_{V_{\kappa}, m}+(1-s) p U_{C_{m}}\right)\left(X^{n}, Y^{n}\right)} p\left|\left(U_{V_{\kappa, m}}-U_{C_{m}}\right)\left(X^{n}, Y^{n}\right)\right|
\end{aligned}
$$

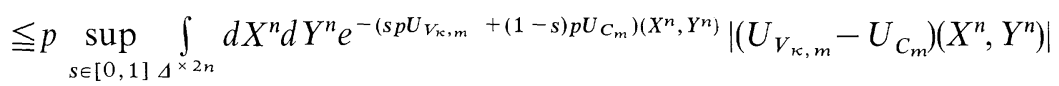

$$
\begin{aligned}
& \leqq p \sup _{s \in[0,1]}\left\|e^{-p U_{V_{\kappa}, m}}\right\|_{1+\delta}^{s}\left\|e^{-p U_{C_{m}}}\right\|_{1+\delta}^{1-s} \times\left\|U_{V_{\kappa, m}}-U_{C_{m}}\right\|_{\frac{\delta}{1+\delta}},
\end{aligned}
$$


by the Hölder inequality, and $\delta>0$ is picked so that $p \alpha(1+\delta)<1$ which is possible, since $p \cdot \alpha<1$. The first and the second factor on the r.h.s. of (3.25) are bounded uniformly in $\kappa<\infty$ and $s \in[0,1]$ by Jensen's inequality - see (3.9) - and (3.22), since $p \alpha(1+\delta)<1$, and the third factor is easily seen to tend to 0 , as $\kappa \rightarrow \infty$. This proves (3.19); (3.20) follows by almost identical arguments. Finally (3.21) follows from the uniform bounds of Corollary 3.5, (1) and (3.20). This completes the proof of (1). Obviously (2) follows from (1) and Corollaries 3.5, 3.6. Q.E.D.

We are now prepared to prove the existence of the thermodynamic limit of the pressure (grand canonical ensemble).

\section{d) Existence of the Thermodynamic Limit}

Theorem 3.8. (Thermodynamic limit for the pressure.)

Let $\alpha<1$, i.e. $\beta \varepsilon^{2}<4 \pi$, (and we set $\left.\beta=\frac{1}{k T}=1\right)$, and $m>0$. Let $\Lambda_{l \times t}$ be an arbitrary rectangle with sides of length $l$ and $t$.

Then for all real $z$

$\lim _{\substack{l \rightarrow \infty \\ t \rightarrow \infty}} \frac{1}{l t} \log \Xi\left(C_{m}, z \chi_{\Lambda_{l \times t}}\right) \equiv p\left(C_{m}, z\right) \quad$ exists and is convex in $z$.

Proof. By Theorem 3.7 and the Markov property of the Gaussian measure $d \mu_{C_{m}}$ (see Section 2, f), Theorem 2.6; [23]) there exists a Hilbert space $\mathscr{H}$ with scalar product $(\cdot, \cdot)$, a vector $\Omega_{0} \in \mathscr{H}$ and (for real $z$ ) a transfer matrix $e^{-t H_{l}(z)}$ generated by a densely defined, selfadjoint, operator $H_{l}(z)$ on $\mathscr{H}$ which is bounded below such that

$$
\begin{aligned}
\left(\Omega_{0}, e^{-t H_{l}(z)} \Omega_{0}\right) & =\Xi\left(C_{m}, z \chi_{\Lambda_{l \times t}}\right)=\Xi\left(C_{m}, z \chi_{\Lambda_{t \times l}}\right) \\
& =\left(\Omega_{0}, e^{-l H_{t}(z)} \Omega_{0}\right) .
\end{aligned}
$$

These equations were first proved by Nelson [24] in the framework of the well known $P(\varphi)_{2}$-models. For a proof see also [28, Chapter 5]. By Theorem 3.7 these results extend trivially to the case at hand.

By the spectral theorem

$$
\left(\Omega_{0}, e^{-t H_{l}(z)} \Omega_{0}\right)=\int e^{-t \lambda} d \varrho_{l, z}(\lambda),
$$

for some probability measure $\varrho_{l, z}$ supported on $[a, \infty)$, where $a=\inf \operatorname{spec} H_{l}(z)>$ $-\infty$.

Let $0<p<1$. Then by the Hölder inequality

$$
\int e^{-p t \lambda} d \varrho_{l, t}(\lambda) \leqq\left(\int e^{-t \lambda} d \varrho_{l, z}(\lambda)\right)^{p} .
$$

Therefore

$$
\frac{1}{p t l} \log \left(\Omega_{0}, e^{-p t H_{l}(z)} \Omega_{0}\right) \leqq \frac{1}{t l} \log \left(\Omega_{0}, e^{-t H_{l}(z)} \Omega_{0}\right)
$$

(by the monotonicity of the logarithm). This inequality proves that $\frac{1}{t l} \log \Xi\left(C_{m}, z \chi_{\Lambda_{l \times t}}\right)$ is monotone increasing in $t$. By (3.26) $t$ and $l$ play symmetric roles which yields monotonicity in $l$, as well. 
Thus $\frac{1}{t l} \log \Xi\left(C_{m}, z \chi_{A_{l \times}}\right)$ is monotone increasing in $t$ and $l$, and by Theorem 3.7, (2) it is uniformly bounded in $t, l$. This proves existence of the limit $p\left(C_{m}, z\right)$. Remark. These arguments are just a rephrasing of arguments due to Guerra [15].

We now prove convexity.

For $0<\lambda<1$

$$
\begin{aligned}
& \Xi\left(C_{m}, \lambda z \chi_{A}\right)=\left\langle\exp \left\{: \cos \varepsilon \Phi:_{C_{m}}\left(\lambda z \chi_{A}\right)\right\}\right\rangle_{C_{m}} \\
& \leqq\left\langle\exp \left\{: \cos \varepsilon \Phi:_{C_{m}}\left(z \chi_{A}\right)\right\}\right\rangle_{C_{m}}^{\lambda}=\Xi\left(C_{m}, z \chi_{A}\right)^{\lambda},
\end{aligned}
$$

by the Hölder inequality applied to $\langle\cdot\rangle_{C_{m}}=\int_{\mathscr{S}^{\prime}} \cdot d \mu_{C_{m}}(\Phi)$. Hence $p\left(C_{m}, \lambda z\right) \leqq \lambda p\left(C_{m}, z\right)$.

Next we apply the Hölder inequality to $e^{\left\{: \cos \delta \Phi: C_{m}\left(\lambda\left(z-z_{0}\right) x_{A}\right)\right\}}$ integrated with the measure

$$
\Xi\left(C_{m}, z_{0} \chi_{A}\right)^{-1} e^{\left\{: \cos \varepsilon \Phi: c_{m}\left(z_{0} \chi_{A}\right)\right\}} d \mu_{C_{m}}(\Phi)
$$

and conclude that

$$
p\left(C_{m},(1-\lambda) z_{0}+\lambda z\right) \leqq(1-\lambda) p\left(C_{m}, z_{0}\right)+\lambda p\left(C_{m}, z\right),
$$

for $\lambda \in[0,1]$ which proves convexity; see also [28, Chapter 6]. Q.E.D.

Remark. For a proof of the existence of the thermodynamic limit of the free energy (canonical ensemble) based on the stability estimates of Corollary 3.6 and Theorem 3.7 we refer to the standard methods; see e.g. [26, Chapter 3], and refs. given there.

It is then an easy exercise which we leave to the reader to show that the canonical and the grand canonical ensemble for our CSM system are equivalent in the sense of [26, Section 3.4]. The proof follows by adapting the arguments of $[26,3.4 .5]$ and using Corollary 3.6.

Theorem 3.9. (Thermodynamic limit for correlation functions.)

Let $\alpha<\frac{4}{\pi^{2}}-$ i.e. $\beta \varepsilon^{2}<\frac{16}{\pi}-$ and $m>0$.

Let $z$ be an arbitrary, real number, $n$ a positive integer, $e_{j}= \pm 1, j=1, \ldots, n$, and $Q^{n}=\left(x_{1}, e_{1}, \ldots, x_{n}, e_{n}\right)$.

Then

(1) The classical correlation functions in the grand canonical ensemble in a region $\Lambda \subset \mathbb{R}^{2}$ are given by

$$
\varrho_{\Lambda}^{z}\left(Q^{n}\right)=z^{n} \Xi\left(C_{m}, z \cdot \chi_{A}\right)^{-1} \times\left\langle\prod_{j=1}^{n}: e^{i e_{\varepsilon} \varepsilon \Phi}:_{C_{m}}\left(x_{j}\right) e^{: \cos \varepsilon \Phi:_{C_{m}}\left(z \chi_{A}\right)}\right\rangle C_{m} .
$$

(2) Let $\left\{\Lambda_{i}\right\}_{i \in I}$ be an increasing family of open regions in $\mathbb{R}^{2}$. Then

$$
\lim _{\Lambda_{\imath} \rightarrow \mathbb{R}^{2}} \varrho_{\Lambda_{\imath}}^{z}\left(Q^{n}\right) \equiv \varrho^{z}\left(Q^{n}\right)
$$


exists in the sense of convergence of measures on $\mathbb{R}^{2 n}$. The correlation functions $\varrho^{z}\left(Q^{n}\right)$ are Euclidean invariant.

(3) There exists a Hausdorff measure space $(X, \Sigma)$ and a regular Borel probability measure $d \mu$ on $(X, \Sigma)$ such that

$$
\varrho^{z}\left(Q^{n}\right)=\int_{X} d \mu(\chi) \varrho_{\chi}^{z}\left(Q^{n}\right), \text { for all } n,
$$

where the measures $\varrho_{\chi}^{z}$ are the pure phase correlation functions; ( $\operatorname{sets}$ in $\Sigma$ correspond to measurable sets of pure phases). For $\mu$-almost all $\chi \in X, \varrho_{\chi}^{z}\left(Q^{n}\right)$ is Euclidean invariant and has the cluster property, (in particular the equilibrium state determined by $\left\{\varrho_{\chi}^{z}\left(Q_{n}\right)\right\}_{n=0}^{\infty}$ is ergodic under the action of the translation group). The $\Phi \mapsto-\Phi$ symmetry is broken in a set $\Delta$ of pure phases of positive $\mu$-measure, meaning that a single particle with charge $\pm \varepsilon$ in a pure phase $\chi \in \Delta$ has a non-vanishing, constant potential energy, provided there exist at least 2 pure phases.

Proof. (1) follows from Lemma 2.2 and [26, Section 4.2].

(2) is proven by noticing that the classical CSM system with two body Yukawa forces is equivalent to a generalized ferromagnetic Ising model with nearest neighbour interactions [16, 28, Chapter VIII] and then using the second Griffiths inequality $[13,16]$ to prove convergence, as $\Lambda \rightarrow \mathbb{R}^{2}$. The complete proof is patterned after the one of Nelson $[16,25]$ of an analogous result in the $P(\varphi)_{2}$ quantum field theory. Additional technical lemmas are required to obtain uniform bounds on the correlation functions $\varrho_{A}^{z}$ and due to the fact that one has to replace the random variables $: e^{i e_{\varepsilon} \varepsilon \Phi}$ : by the field $\Phi$ to make Nelson's argument work. For a complete proof of (2) the reader is referred to [8].

(3) follows from (2) by applying the isomorphism $\tilde{I}_{1}$ and a general theorem about the decomposition into pure phases proved in $[10,11]$. Q.E.D.

Remarks. 1) Obviously, for $\beta \varepsilon^{2} \geqq 4 \pi, e^{-\beta U_{C_{m}}\left(X^{n}, Y^{n}\right)}$ is not integrable with respect to $d X^{n} d Y^{n}$ which is trivially checked for $n=1 ; \Xi\left(C_{m}, z \cdot \chi_{\Lambda}\right)=\infty$ for $z \neq 0, \Lambda \neq 0$, and the correlation functions $\varrho_{\Lambda}^{z}\left(Q^{n}\right)$ do not exist. This is the collapse catastrophe (formation of pairs) described in the introduction.

The more severe restriction, namely $\beta \varepsilon^{2}<\frac{16}{\pi}$ rather than $\beta \varepsilon^{2}<4 \pi$, required in Theorem 3.9 (in contradistinction to $\beta \varepsilon^{2}<4 \pi$ required in all previous results of Section 3) is presumably an artefact of non-optimal estimates; see [8].

2) For $\beta \varepsilon^{2}<4 \pi$ and $m>0$ the correlation function $\varrho^{z}\left(Q^{n}\right)$ are analytic in $z$ for $\left|z / m^{2}\right|<r_{\beta \varepsilon^{2}}$ (with $r_{\beta \varepsilon^{2}}>0$ for $\beta \varepsilon^{2}<4 \pi$ ) and they have exponential cluster properties; (the interval $0<z<m^{2} r_{\beta \varepsilon^{2}}$ is thus contained in the single phase region). In addition to the chemical potential for the number of particles $(-\log z)$ one can also introduce a chemical potential $\mu$ for the charge. If $\left|z / m^{2}\right|$ and $e^{-\mu}$ are small the correlation functions still exist and have exponential cluster properties. These results are based on the Glimm-Jaffe-Spencer cluster expansion; see their contribution in [7] and refs. given there. The proof is given in [9].

3) Applying the isomorphisms $\tilde{I}_{1}$ and $\tilde{I}_{2}$ described in the introduction Theorem 3.9 establishes existence and Poincaré-covariance of the quantum field theories, (the Sine-Gordon equation with a mass term and Quantum Electrodynamics + Thirring interaction in two space-time dimensions), listed in $\tilde{B}$ and $\tilde{C}$. 


\section{Stability and the Thermodynamic Limit for the Coulomb Potential}

In this section we establish all the results of Section 3 for two component, neutral CSM systems with two body Coulomb potentials with one proviso: A precise analogue of Theorem 3.9 can only be proven under the assumption that Debye screening forces the classical, neutral Coulomb system in the thermodynamic limit to have a finite correlation length - i.e. no long range order. (Without this assumption one can still prove existence of correlation functions in the thermodynamic limit but their uniqueness is then an open problem; see [8].) Applying the isomorphisms $I_{1}, I_{2}$ described in the introduction our results in Section 4 imply existence and Poincare covariance of the field theories defined in $B$ and $C$ - the quantum Sine-Gordon equation and the massive Thirring model (in the vacuum sector); see [8].

a) Approximating and Estimating the Coulomb-by the Yukawa CSM System We define

$$
V_{m}(x-y) \equiv C_{m}(x-y)+\frac{1}{4 \pi} \ln \left(\mathrm{cm}^{2}\right),
$$

where $c$ is a numerical constant, and

$$
\begin{aligned}
V_{0}(x-y) & \equiv-\frac{1}{4 \pi} \ln |x-y|^{2} \\
& =\frac{1}{2 \pi} \ln \frac{1}{|x-y|} .
\end{aligned}
$$

It is easy to prove that

$$
\lim _{x \rightarrow 0}\left[V_{m_{1}}(x)-V_{m_{2}}(x)\right]=0 ;
$$

this is well known; see e.g. [2, Formula 2.7].

Lemma 4.1. Let $\beta=1, \varepsilon^{2}<4 \pi$ and $\varepsilon^{2}<p \varepsilon^{2}<4 \pi$. Let $g$ be a positive function in $L^{p^{\prime}}\left(\Delta, d^{2} x\right)$, for some square $\Delta$ of finite volume.

Then for all $0<m \leqq 1$

$$
Z_{n}\left(V_{m}, g\right) \leqq Z_{n}\left(V_{1}, g\right)=c^{\frac{n \varepsilon^{2}}{4 \pi}} Z_{n}\left(C_{1}, g\right) \text {. }
$$

Proof. This is Lemma 2.1, i.e.

$$
\begin{aligned}
Z_{n}\left(V_{m}, g\right) & =\int d X^{n} d Y^{n} g\left(X^{n}\right) g\left(Y^{n}\right) e^{-U_{V_{m}}\left(X^{n}, Y^{n}\right)} \\
& =\int d X^{n} d Y^{n} g\left(X^{n}\right) g\left(Y^{n}\right) e^{-U_{V_{1}}\left(X^{n}, Y^{n}\right)} \cdot e^{-\int d^{2} x d^{2} y \varrho_{n}(x)\left(V_{m}-V_{1}\right)(x-y) \varrho_{n}(y)},
\end{aligned}
$$

where $\varrho_{n}(x)=\sum_{j=1}^{n} \varepsilon\left\{\delta\left(x-x_{j}\right)-\delta\left(x-y_{j}\right)\right\}$, and by (4.3) the constant occurring in Lemma 2.1, (2.9) is equal to 0 . The existence of $\int d x d y \varrho_{n}(x)\left(V_{m}-V_{1}\right)(x-y) \varrho_{n}(y)$ is 
obvious. Since $\int_{\Delta} d x \varrho_{n}(x)=0-$ neutrality -

$$
\begin{aligned}
& \int d^{2} x d^{2} y \varrho_{n}(x)\left(V_{m}-V_{1}\right)(x-y) \varrho_{n}(y) \\
& \quad=\int d^{2} x d^{2} y \varrho_{n}(x)\left(C_{m}-C_{1}\right)(x-y) \varrho_{n}(y) \\
& \quad=\int d^{2} k\left|\varrho_{n}(k)\right|^{2}\left(\frac{1}{k^{2}+m^{2}}-\frac{1}{k^{2}+1}\right) \geqq 0 .
\end{aligned}
$$

The lemma follows from (4.5) and (4.6). Q.E.D.

Lemma 4.2. Let $e_{j}= \pm 1, j=1, \ldots, n$. Under the assumptions of Lemma 4.1

(1) $\lim _{m \rightarrow 0}\left\langle\prod_{j=1}^{n}: e^{i e_{j} \varepsilon \Phi}:_{V_{1}}\left(x_{j}\right)\right\rangle_{V_{m}}=\lim _{m \rightarrow 0} m^{\frac{n \varepsilon^{2}}{4 \pi}}\left\langle\prod_{j=1}^{n}: e^{i e_{j} \varepsilon \Phi}:_{V_{m}}\left(x_{j}\right)\right\rangle_{V_{m}}$

$$
=\left\{\begin{array}{l}
0 \text { if } \sum_{j=1}^{n} e_{j} \neq 0 \quad \text { (in particular if } n \text { is odd) } \\
e^{-U\left(Q^{n}\right)}, \text { if } \sum_{j=1}^{n} e_{j}=0
\end{array}\right.
$$

where $Q_{n}=\left(x_{1}, e_{1}, \ldots, x_{n}, e_{n}\right)$, and $U\left(Q_{n}\right)=\frac{\varepsilon^{2}}{2 \pi} \sum_{1 \leqq i<j \leqq n} e_{i} e_{j} \ln \frac{1}{\left|x_{i}-x_{j}\right|}$.

(2) $\lim _{m \rightarrow 0} \sum_{q=0}^{n}\left(\begin{array}{l}\mathrm{n} \\ q\end{array}\right) Z_{n}^{2 q-n}\left(V_{m}, g\right)=2^{n} \lim _{m \rightarrow 0}\left\langle\left\{: \cos \varepsilon \Phi:_{V_{1}}(g)\right\}^{n}\right\rangle_{V_{m}}$

$$
=\left\{\begin{array}{l}
0 \text { if } n \text { is odd } \\
\left(\begin{array}{c}
n \\
n / 2
\end{array}\right) Z_{n / 2}\left(V_{0}, g\right) \quad \text { if } n \text { is even, and in this case } \\
Z_{n / 2}\left(V_{0}, g\right)=\lim _{m \rightarrow 0} Z_{n / 2}\left(V_{m}, g\right) .
\end{array}\right.
$$

(3) $Z_{n}\left(V_{0}, g\right) \leqq Z_{n}\left(V_{1}, g\right)$.

Proof. Part (1) of this lemma is well known from the theory of the free, massless quantum Klein-Gordon field in two space-time dimensions. It is discussed e.g. in $[29,2]$. The proof follows directly from Lemma 2.2, (2.17), (2.16), and (4.1), (4.2). Part (2) is an immediate consequence of formulas (2.21), (2.18), and of Lemma 4.2, (1). Finally (3) follows from (2) and Lemma 4.1. Q.E.D.

\section{Corollary 4.3.}

$\lim _{m \rightarrow 0} \Xi\left(V_{m}, 2 z g\right)=Z\left(V_{0}, z g\right) \equiv \Xi\left(V_{0}, 2 g\right)$.

Proof. The corollary is a consequence of Lemma 4.2, (2), of (4.4) and Lemma 4.2 , (3) and of the bounds established in Corollary 3.5, Theorem 3.7 which guarantee that the power series expansion of $\Xi\left(V_{1}, 2 z g\right)$ in $z$ converges absolutely for all $z \in \mathbb{C}$. Q.E.D. 
Theorem 4.4. (Stability of the classical Coulomb system.) Under the assumptions of Lemma 4.1 and Corollary 3.5,(2)

$$
\begin{gathered}
\left|\Xi\left(V_{0}, z \chi_{A}\right)\right| \leqq K_{p} e^{\bar{K}_{p}|\operatorname{Re} z|^{\mid p / p-1}|\Lambda|} \\
\left|Z_{n}\left(V_{0}, z \chi_{A}\right)\right| \leqq K_{p}^{n}|z|^{2 n}\left(\frac{n}{|\Lambda|}\right)^{\frac{1-p_{n}}{p}}(n !)^{2},
\end{gathered}
$$

where $K_{p}, \bar{K}_{p}$ are constants which are finite for $\varepsilon^{2}<p \varepsilon^{2}<4 \pi$.

Proof. This follows from Lemma 4.2, (2) and (3), from Corollary 4.3 and from the bounds proved in Corollaries 3.5, 3.6, Theorem 3.7. Q.E.D.

\section{b) Existence of the Thermodynamic Limit}

Theorem 4.5. (Thermodynamic limit of the pressure.)

Let $\varepsilon^{2}<4 \pi$ and $\left\{\Lambda_{\times x}\right\}$ a family of rectangles such as in Theorem 3.8. Then for all real $z$

$$
\lim _{\substack{l \rightarrow \infty \\ t \rightarrow \infty}} \frac{1}{l t} \log \Xi\left(V_{0}, z \chi_{\Lambda_{l} \times}\right) \equiv p\left(V_{0}, z\right) \text { exists and is convex in } z \text {. }
$$

Proof. This is the analogue of Theorem 3.8 and follows from Theorem 4.4, Corollary 4.3 and the arguments used in the proof of Theorem 3.8 (i.e. monotonicity in $l$ and $t$ ). Q.E.D.

Remark. For a direct proof of the existence of the thermodynamic limit of the free energy (canonical ensemble) we refer the reader to the techniques of [19] which apply to the case considered here. The equivalence of the canonical and the grand canonical ensemble remains true. (See also (4.16) below.)

Definition. Let $\varrho_{m}^{z}\left(Q^{n}\right)$ be the classical correlation functions in the thermodynamic limit associated with Yukawa two body forces with potential $V_{m}$. Here $Q^{n}=\left(x_{1}, e_{1}, \ldots, x_{n}, e_{n}\right) ; e_{j}= \pm 1$, for $j=1, \ldots, n$. (These correlation functions were constructed in Theorem 3.9 for all $m>0$, arbitrary, real $z$ and $\left.\varepsilon^{2}<\frac{16}{\pi}\right)$.

Let $z$ be a positive number. We define

$$
\xi(m, z)^{-1}=\lim _{|x| \rightarrow \infty}-\frac{1}{|x|} \log \left\{\varrho_{m}^{z}(x, 1,0,-1)-\varrho_{m}^{z}(0,1)^{2}\right\} ;
$$

$\xi(m, z)$ is the correlation length. As a consequence of correlation inequalities [16] one can show that $\xi(m, z)$ increases, as $m$ decreases; see [8].

Theorem 4.6. (Existence of the $m=0$ correlation functions.)

Let $\varepsilon^{2}<\frac{16}{\pi}$ and suppose that

$$
\xi(0, z) \equiv \sup \xi(m, z)<\infty \text {. }
$$


Then for all positive integers $n$

$$
\lim _{m \rightarrow 0} \varrho_{m}^{z}\left(Q^{n}\right) \equiv \varrho_{0}^{z}\left(Q^{n}\right)
$$

exists in the sense of measures on $\mathbb{R}^{2 n}$; the measures $\left\{\varrho_{0}^{z}\left(Q^{n}\right)\right\}_{n=0}^{\infty}$ are Euclidean invariant and have exponential cluster properties (with decay rate $\left.\xi(0, z)^{-1}\right)$.

Remarks. This theorem is a consequence of correlation inequalities $[16,8]$ of the type of the second Griffiths inequality and uniform bounds on the measures $\left\{\varrho_{m}^{z}\left(Q^{n}\right)\right\}_{m>0}$ in $m$. It is proved in [8], where two equivalent constructions of the correlation functions $\varrho_{0}^{z}\left(Q^{n}\right)$ are given, one of them being the one described above. Debye screening and the isomorphism between the classical, neutral Coulomb gas and the massive Thirring model suggest that $\xi(0, z)$ is finite for some values of $z$ (see also [3]). If this is true then $\xi(0, z)<\infty$, for all $z \neq 0$ which we prove belov. (Since $z$ determines the average density $\varrho$ and $\sqrt[3]{\varrho^{-1}}$ has the dimension of a length the conjecture $\xi(0, z)<\infty$ is certainly not absurd.)

A weaker form of Theorem 4.6 which is not based on the conjecture (4.7) is proved in [8]. In this case the uniqueness of $\varrho_{0}^{z}\left(Q^{n}\right)$ is however unknown.

We now explore the scaling behaviour of the canonical partition function of the classical, neutral Coulomb gas in order to derive some explicit expressions for the pressure $p$ and the correlation length $\xi$ as functions of the fugacity $z$ and to rigorously prove the well known equation of state $[21,17]$

$$
p=\frac{\varrho}{\beta}\left(1-\frac{\beta \varepsilon^{2}}{8 \pi}\right) .
$$

Let $\alpha \equiv \frac{\beta \varepsilon^{2}}{4 \pi}$ (and we set $\beta=1$ in the following); let $\left.p(\alpha, z) \equiv p\left(V_{0}, z\right)\right|_{\varepsilon^{2}=4 \pi \alpha \text {. We }}$. first prove that the pressure $p(\alpha, z)$ in the grand canonical ensemble has the form

$$
p(\alpha, z)=F(\alpha) z^{\frac{2}{2-\alpha}} .
$$

By Theorem 4.5

$$
p(\alpha, z)=\lim _{\substack{l \rightarrow \infty \\ t \rightarrow \infty}} \frac{1}{l \cdot t} \log \Xi\left(\alpha, z \chi_{\Lambda_{l \times}}\right),
$$

where $\left.\Xi\left(\alpha, z \chi_{\Lambda_{l \times t}}\right) \equiv \Xi\left(V_{0}, z \chi_{\Lambda_{l \times t}}\right)\right|_{\varepsilon^{2}=4 \pi \alpha}$ is the grand canonical partition function for a rectangle with sides of length $l$ and $t$. From Theorem 4.5 we know that $p(\alpha, z)$ is a non-negative, convex function of $z$.

By definition

$$
\Xi\left(\alpha, z \chi_{\Lambda_{l \times t}}\right)=\sum_{n=0}^{\infty} \frac{z^{2 n}}{(n !)^{2}} Z_{n}\left(\alpha, \chi_{\Lambda_{l \times t}}\right)
$$

and

$$
Z_{n}\left(\alpha, \chi_{\Lambda_{l \times}}\right)=\int_{\left(A_{l \times t}\right)^{\times 2 n}} \prod_{j=1}^{n} d^{2} x_{j} d^{2} y_{j} \frac{\prod_{1 \leqq i<j \leqq n}\left|x_{i}-x_{j}\right|^{2 \alpha}\left|y_{i}-y_{j}\right|^{2 \alpha}}{\prod_{i, j=1}^{n}\left|x_{i}-y_{j}\right|^{2 \alpha}} .
$$


We now change variables:

We let $l=\lambda l_{0}, t=\lambda t_{0}$, and

$$
\begin{aligned}
& x_{i} \mapsto \bar{x}_{i}=\lambda^{-1} x_{i}, \quad d^{2} x_{i} \mapsto \lambda^{2} d^{2} \bar{x}_{i} \\
& y_{i} \mapsto \bar{y}_{i}=\lambda^{-1} y_{i}, \quad d^{2} y_{i} \mapsto \lambda^{2} d^{2} \bar{y}_{i}, \quad \Lambda_{\lambda l_{0} \times \lambda t_{0}} \mapsto \Lambda_{l_{0} \times t_{0}} .
\end{aligned}
$$

Then by (4.10)

$$
Z_{n}\left(\alpha, \chi_{\Lambda_{\lambda l_{0} \times \lambda t_{0}}}\right)=\lambda^{(2-\alpha) 2 n} Z_{n}\left(\alpha, \chi_{\Lambda_{l_{0} \times t_{0}}}\right)
$$

and hence

$$
\Xi\left(\alpha, z \chi_{\Lambda_{\lambda l_{0} \times \lambda t_{0}}}\right)=\Xi\left(\alpha, \lambda^{2-\alpha} z \chi_{\Lambda_{l \times t}}\right) \text {. }
$$

By (4.9) and (4.12)

$$
\begin{aligned}
\lambda^{2} p(\alpha, z)= & \lambda^{2} \lim _{\substack{l_{0} \rightarrow \infty \\
t_{0} \rightarrow \infty}} \frac{1}{\lambda l_{0} \lambda t_{0}} \log \Xi\left(\alpha, z \chi_{\Lambda_{\lambda l_{0} \times \lambda t_{0}}}\right) \\
= & \lim _{\substack{l_{0} \rightarrow \infty \\
t_{0} \rightarrow \infty}} \frac{1}{l_{0} \cdot t_{0}} \log \Xi\left(\alpha, \lambda^{2-\alpha} z \chi_{\Lambda_{l_{0} \times t_{0}}}\right) \\
= & p\left(\alpha, \lambda^{2-\alpha} z\right) .
\end{aligned}
$$

The non-negativity and convexity of $p(\alpha, z)$ as a function of $z$ combined with (4.13) now yield

Theorem 4.7. (1) $p(\alpha, z)=F(\alpha) z^{\frac{2}{2-\alpha}}$, for some positive function $F(\alpha)$ which is finite for $\alpha<1$. In the variable $\mu=\ln z$ (chemical potential)

$$
p\left(\alpha, e^{\mu}\right) \equiv \tilde{p}(\alpha, \mu)=F(\alpha) e^{\frac{2}{2-\alpha} \mu} .
$$

(2) For $\alpha<1$ the neutral, classical Coulomb gas has no phase transitions as one varies the density $\varrho$ of the gas on $(0, \infty)$.

Proof. We have already proved (1); (2) is a direct consequence of (1). For the convenience of the reader we compute the Legendre transform of $\tilde{p}(\alpha, \mu)$ with respect to $\mu$, i.e. the free energy $f(\alpha, \varrho)$ as a function of the density $\varrho$ of the gas:

We get

$$
\varrho \equiv \frac{\partial \tilde{p}(\alpha, \mu)}{\partial \mu}=\frac{2}{2-\alpha} F(\alpha) e^{\frac{2}{2-\alpha} \mu},
$$

i.e.

$$
\mu(\varrho)=\frac{2-\alpha}{2} \ln \left[\frac{(2-\alpha) \varrho}{2 F(\alpha)}\right],
$$

and

$$
\begin{aligned}
f(\alpha, \varrho) & \equiv \mu(\varrho) \cdot \varrho-\tilde{p}(\alpha, \mu(\varrho)) \\
& =\frac{2-\alpha}{2} \varrho\left[\ln \left[\frac{(2-\alpha) \varrho}{2 F(\alpha)}\right]-1\right] .
\end{aligned}
$$


Since $F(\alpha)$ is positive $f(\alpha, \varrho)$ is analytic in $\varrho$ on $(0, \infty)$ which is interpreted as absence of phase transitions as one varies $\varrho$. Q.E.D.

Notice that

$$
\begin{aligned}
\tilde{p}(\alpha, \mu(\varrho)) & =\varrho\left(1-\frac{\alpha}{2}\right) \\
& =\varrho\left(1-\frac{\varepsilon^{2}}{8 \pi}\right) .
\end{aligned}
$$

This is the equation of state of the classical, neutral Coulomb gas. It was earlier derived in [21,17], where, however, (4.9) remained an unproven assumption. Theorem 4.7, (1) shows that for the Coulomb gas an expansion of the pressure $p(\alpha, z)$ in powers of $z$ is impossible (it is easy to see that the explicit expressions for the coefficients of such an expansion are infrared divergent).

Next we want to derive an explicit expression for the correlation length $\xi(m, z)$ when $m=0$. Let $\alpha$ be fixed. For the following we must assume that there exists some $z_{0} \neq 0$ such that

$$
\left.\sup _{m>0} \xi\left(m, z_{0}\right)\right|_{\mathcal{\varepsilon}^{2}=4 \pi \alpha}<\infty .
$$

It is convenient to change notations:

$\left.\begin{array}{rl} & \left.\xi(\alpha, z) \equiv \xi(m, z)\right|_{m=0, \varepsilon^{2}=4 \pi \alpha} . \\ \text { We let } & u_{1}^{z} \equiv \varrho_{m=0}^{z}(x, 1)=\varrho_{m=0}^{z}(0, \pm 1), \\ \text { and } & u_{2}^{z}(x) \equiv \varrho_{m=0}^{z}(x, 1,0,-1)-\left(u_{1}^{z}\right)^{2} .\end{array}\right\}$

Using a slight generalization of the scaling relations (4.13) proved under the hypothesis (4.16) in [8] one can show

$$
\lambda^{2 \alpha} u_{2}^{\lambda \alpha-2 z_{0}}(x)=u_{2}^{z_{0}}\left(\lambda^{-1} x\right) .
$$

Similar, explicit scaling - ("Callan-Symanzik"-) equations can be derived for the $n$-point Ursell functions $u_{n}^{z}\left(x_{1}, \ldots, x_{n-1}\right)$, for all $n$; see [8]. We do not use them in this paper.

It is well known that

$$
\xi\left(\alpha, z_{0}\right)^{-1}=\lim _{|x| \rightarrow \infty}-\frac{1}{|x|} \log u_{2}^{z_{0}}(x),
$$

see e.g. [8].

Thus, by (4.18)

$$
\begin{aligned}
\xi\left(\lambda^{\alpha-2} z_{0}, \alpha\right)^{-1} & =\lim _{|x| \rightarrow \infty}-\frac{1}{|x|} \log u_{2}^{\lambda^{\alpha-2} z_{0}}(x) \\
& =\lim _{|x| \rightarrow \infty}-\frac{1}{|x|} \log \left[\lambda^{2 \alpha} u_{2}^{\lambda^{\alpha-2} z_{0}}(x)\right] \\
& =\lambda^{-1} \lim _{|x| \rightarrow \infty}-\frac{1}{\lambda^{-1}|x|} \log u_{2}^{z_{0}}\left(\lambda^{-1} x\right) \\
& =\lambda^{-1} \xi\left(\alpha, z_{0}\right), \text { for arbitrary } \lambda>0 .
\end{aligned}
$$


Therefore

$$
\xi\left(\lambda z_{0}, \alpha\right)=G(\alpha)\left(\lambda z_{0}\right)^{\frac{1}{\alpha-2}} \text { or } \xi(z, \alpha)=G(\alpha) z^{\frac{1}{\alpha-2}},
$$

for some measurable function $G$ of $\alpha$.

We remark that $G(\alpha=0)=0$. Continuity of $G$ near $\alpha=0$ would imply that $\xi(z, \alpha)$ is finite for all $z \neq 0$, provided $\alpha$ is sufficiently small. A Lee-Yang type theorem in $\alpha$ would imply that $\xi(z, \alpha)$ is finite for all $z \neq 0$ and all but at most countably many $\alpha \in[0,1)$. We have not yet been able to prove either of these conjectures; (a proof may require some sort of "large $z$-expansion"). Certainly Eq. (4.20) is consistent with our conjecture of Debye-screening and makes it more precise.

\section{c) Pair Condensation as $T \searrow T_{c}$}

Here we construct a renormalized limit for the pressure, as $\alpha \gg 1$ (i.e. $T \searrow T_{c}$ ) using some preliminary estimates of [4]. Let $\Delta$ be a square of area $\frac{1}{4}$ in $\mathbb{R}^{2}$.

We define $z(\alpha) \equiv Z_{1}\left(\alpha, \chi_{\Delta}\right)^{-\frac{1}{2}}$.

Lemma 4.8. There exists a finite constant $c_{1} \geqq 1$ such that

$\limsup _{\alpha>1} p(\alpha, z(\alpha) \cdot z)=c_{1} z^{2}$.

Proof. (1) Because of scaling - Theorem 4.7 - it suffices to prove (4.21) for small $|z|$. It then extends to arbitrary $z$, by Theorem $4.7,(1)$.

(2) Therefore we only must prove that

$$
\left|\Xi\left(\alpha, z(\alpha) z \chi_{\Lambda}\right)\right|<K e^{\bar{K}|\Lambda|},
$$

for all $\alpha<1$ and sufficiently small $z$, with constants $K$ and $\bar{K}$ independent of $\alpha$.

By Lemma 4.2, (3)

$$
\begin{aligned}
\Xi\left(\alpha, z(\alpha) z \chi_{\Lambda}\right) & \leqq\left.\Xi\left(V_{1}, z(\alpha) z \chi_{\Lambda}\right)\right|_{\varepsilon^{2}=4 \pi \alpha} \\
& =\left.\Xi\left(C_{1}, z(\alpha) c^{2} z \chi_{\Lambda}\right)\right|_{\varepsilon^{2}=4 \pi \alpha}
\end{aligned}
$$

where $c$ is the constant defined in (4.1).

By Theorem 2.5 and Corollary 3.2

$$
\begin{aligned}
& \left.\Xi\left(C_{1}, z(\alpha) c^{\frac{\alpha}{2}} z \chi_{\Lambda}\right)\right|_{\varepsilon^{2}=4 \pi \alpha} \\
& \leqq \prod_{\Delta \cap A \neq \emptyset}\left[\left.\Xi\left(C_{1}, z(\alpha) p c^{\frac{\alpha}{2}} z \chi_{\Delta}\right)\right|_{\varepsilon^{2}=4 \pi \alpha}\right]^{\frac{1}{p}} \\
& \leqq\left[\left.\Xi\left(C_{0}^{S}, z(\alpha) r p c^{\frac{\alpha}{2}} z w \chi_{\Delta_{\frac{1}{2}}}\right)\right|_{\varepsilon^{2}=4 \pi \alpha}\right]^{\frac{K \cdot|A|}{p \cdot r}} \\
& \leqq\left[\left.\Xi\left(C_{0}^{S}, z(\alpha) \bar{z} \chi_{\Delta_{\frac{1}{2}}}\right)\right|_{\varepsilon^{2}=4 \pi \alpha}\right]^{\frac{K \cdot|\Lambda|}{p \cdot r}},
\end{aligned}
$$

where $\Delta_{\frac{1}{2}}$ is the square introduced in Section 3, (a), $C_{0}^{S}$ is defined in Section 3, (b), and

$$
\bar{z}=r \cdot p \cdot|z| \cdot \max \left(c^{\frac{1}{2}}, 1\right) \cdot \sup _{x \in \Delta_{\frac{1}{2}}}|w(x)| .
$$


Thus we are left with estimating $\left.\Xi\left(C_{0}^{S}, z(\alpha) \bar{z} \chi_{\Delta_{\frac{1}{2}}}\right)\right|_{\varepsilon^{2}=4 \pi \alpha}$, uniformly in $\alpha<1$.

(3) This estimate can be reduced to

$$
\left.z(\alpha)^{2 n} Z_{n}\left(C_{0}^{S}, \chi_{\Delta_{\frac{1}{2}}}\right)\right|_{\varepsilon^{2}=4 \pi \alpha} \leqq K_{1}^{n}(n !)^{2},
$$

for all $\alpha<1$, with $K_{1}$ a constant independent of $\alpha$. For the proof of estimate (4.23) we use (3.8) and (3.11)-(3.13) to derive (our notations are as in Theorem 3.4):

$$
\begin{aligned}
& \int_{\substack{\frac{x}{2} 2 n \\
\frac{1}{2}}} d Z^{n} d W^{n}\left|\operatorname{Det}\left(\frac{1}{z_{i}-w_{j}}\right)\right|^{\alpha} \prod_{i=1}^{n}\left|z_{j}-w_{j+n}\right|^{\alpha}\left|w_{j}-z_{j+n}\right|^{\alpha} \\
& \leqq K_{2}^{n} \int_{\Delta_{\frac{1}{2} 2 n}} d Z^{n} d W^{n}\left|\sum_{\pi \in \gamma_{2 n}} \prod_{i=1}^{2 n} \frac{1}{z_{i}-w_{\pi(i)}}\right|^{\alpha} \\
& \leqq K_{2}^{n}\left[\sum_{\pi \in \gamma_{2 n}} \int_{\Delta_{\frac{1}{2}}^{\times} 2 n} d Z^{n} d W^{n} \prod_{i=1}^{2 n} \frac{1}{\left|z_{i}-w_{\pi(i)}\right|^{\alpha}}\right] \\
& \leqq K_{3}^{n}(2 n !) \int_{\Delta_{\frac{x}{2} 2 n}} d Z^{n} d W^{n} \prod_{i=1}^{n} \frac{1}{\left|z_{i}-w_{i}\right|^{\alpha}\left|z_{i+n}-w_{i+n}\right|^{\alpha}} \\
& \leqq(n !)^{2} K_{1}^{n} z(\alpha)^{-2 n}, \quad \text { which proves }(4.23) .
\end{aligned}
$$

See $[4$, Section 3$]$ for related estimates.

Thus

$$
\begin{aligned}
1 & \leqq\left.\Xi\left(C_{0}^{S}, z(\alpha) \bar{z} \chi_{\Delta_{\frac{1}{2}}}\right)\right|_{\varepsilon^{2}=4 \pi \alpha} \\
& \left.\equiv \sum_{n=0}^{\infty} \frac{1}{(n !)^{2}} \bar{z}^{2 n} z(\alpha)^{2 n} Z_{n}\left(C_{0}^{S}, \chi_{\Delta_{\frac{1}{2}}}\right)\right|_{\varepsilon^{2}=4 \pi \alpha} \\
& \leqq \sum_{n=0}^{\infty}\left(K_{1} \bar{z}^{2}\right)^{n} .
\end{aligned}
$$

Since $\bar{z} \propto|z|$, the r.h.s. of (4.24) converges provided $|z|$ is sufficiently small. This completes the proof of (4.22), and (1) and the next result - which shows $c_{1} \geqq 1-$ yield Lemma 4.8. Q.E.D.

\section{Theorem 4.9.}

$\liminf _{\alpha \rightarrow 1} p(\alpha, z(\alpha) z)=z^{2}$.

Proof. Let

$$
\Delta_{\lambda} \equiv\left\{x \mid \frac{x}{\lambda} \in \Delta_{\frac{1}{2}}\right\} \text {. }
$$

1) $\liminf _{\alpha \rightarrow 1} p(\alpha, z(\alpha) z)=\liminf _{\alpha \rightarrow 1} \lim _{\lambda \rightarrow \infty} \frac{1}{\lambda^{2}} \log \Xi\left(\alpha, z(\alpha) z \chi_{\Delta \lambda}\right]$.

\section{Clearly}

$$
\inf _{1>\beta \geqq \alpha} \frac{1}{\lambda^{2}} \log \Xi\left(\beta, z(\beta) z \chi_{\Delta_{\lambda}}\right)
$$

is increasing (more precisely non-decreasing) in $\alpha$ (trivially) and in $\lambda$ by Theorems 4.5 and 3.8 . 
Therefore

$$
\begin{aligned}
& \liminf _{\alpha \rightarrow 1}\left[\lim _{\lambda \rightarrow \infty} \frac{1}{\lambda^{2}} \log \Xi\left(\alpha, z(\alpha) z \chi_{\Delta_{\lambda}}\right)\right] \\
& =\lim _{\lambda \rightarrow \infty} \frac{1}{\lambda^{2}}\left[\liminf \log \Xi\left(\alpha, z(\alpha) z \chi_{\Delta_{\lambda}}\right)\right] .
\end{aligned}
$$

2) From (the proof of) Theorem 4.7 we get

$$
\begin{aligned}
\Xi\left(\alpha, z(\alpha) z \chi_{\Delta_{\lambda}}\right) & \equiv \sum_{n=0}^{\infty} \frac{z^{2 n} z(\alpha)^{2 n}}{(n !)^{2}} Z_{n}\left(\alpha, \chi_{\Delta_{\lambda}}\right) \\
& =\sum_{n=0}^{\infty} \frac{z^{2 n}}{(n !)^{2}} \lambda^{(2-\alpha) 2 n} z(\alpha)^{2 n} Z_{n}\left(\alpha, \chi_{\Delta_{\frac{1}{2}}}\right) .
\end{aligned}
$$

3) It suffices to prove Theorem 4.9 for small $z$. We may let $z$ be so small that (4.22) and (4.24) apply.

It then suffices to show that

$$
\lim _{\alpha \rightarrow 1} z(\alpha)^{2 n} Z_{n}\left(\alpha, \chi_{\Delta_{\frac{1}{2}}}\right)=n \text { ! }
$$

This is proved in [4, Section 3].

Thus

$$
\lim _{\alpha>1} \Xi\left(\alpha, z(\alpha) z \chi_{\Delta_{\lambda}}\right)=\sum_{n=0}^{\infty} \frac{\left(\lambda^{2} z^{2}\right)^{n}}{n !}=e^{\lambda^{2} z^{2}},
$$

and hence

$$
\frac{1}{\lambda^{2}} \lim _{\alpha \rightarrow 1} \log \Xi\left(\alpha, z(\alpha) z \chi_{\Delta \lambda}\right)=z^{2}
$$

which completes the proof of Theorem 4.9 .

\section{Interpretation}

Lemma 4.8 and Theorem 4.9 tell us that as $\alpha \gg 1$, i.e. $T \searrow T_{c}$, the contributions of interactions between pairs of particles of opposite charge are dominant. This is interpreted as a pair condensation process [4]. This interpretation is rigorous if the constant $c_{1}$ in Lemma 4.8 is equal to 1 ; (a proof would require additional estimates). Physically the correct pressure of the classical Coulomb gas for $T<T_{c}$ is then $p(\alpha, z)=z^{2}$ (up to a factor associated with the kinetic energy of particle pairs); $T_{c}$ has the significance of a critical temperature.

The renormalization procedure for the classical, neutral Coulomb gas described in Lemma 4.8 and Theorem 4.9 is certainly rather artificial. What these results really suggest is:

- The classical description is only valid for $T \gg T_{c}$.

- For $T \gtrsim T_{c}$ quantum effects are important.

- The quantum Coulomb gas presumably has a phase transition (associated with pair condensation) at $T \approx T_{c}$ (a rigorous proof has not been found). 
Obviously Lemma 4.8 and Theorem 4.9 can be extended to the classical Yukawa gas $(m>0)$, (at least for small enough $|z|)$. Furthermore the methods and results of Sections 3 and 4, a) and b) can be adapted to one dimension.

Our results in Section 4, b) and c), in particular Theorem 4.7, (1) and (4.15), suggest some interesting conjectures for the field theories described in the introduction under $B$ and $C$ :

For $1<\frac{\varepsilon^{2}}{4 \pi}<2$ the field theories $B, C$ are probably renormalizable; see also [2]. It follows from Theorem 4.7, (1) that they are not superrenormalizable. For $\varepsilon^{2}=4 \pi$ they are known to be superrenormalizable and to be equivalent to a free Dirac field of mass $z$; see [2]. For further discussion see $[8,9]$.

\section{Stability of the Quantum Yukawa- and Coulomb System}

Physically this section is possibly the most interesting one; it is certainly the shortest one. We prove that the quantum system consisting of infinitely many non-relativistic fermions of arbitrary spin and charge $\pm \varepsilon$ interacting via a two body Yukawa- or Coulomb force is stable. The thermodynamic limit of the free energy or the pressure can then be constructed in a standard manner - in the case of Coulomb forces one uses the powerful methods of Lieb and Lebowitz [19].

The basic idea for the proof of stability is the following: Use the GoldenThompson inequality $\left(\operatorname{Tr}\left(e^{A+B}\right) \leqq \operatorname{Tr}\left(e^{A} e^{B}\right)\right)$ and Fermi statistics (which when combined with Hadamard's inequality for determinants gives the Gibbs factor $\left.\frac{1}{(n !)^{2}}\right)$ to reduce the stability estimate for the quantum systems to the one for the classical systems established in Sections 3 and 4.

It should not be very difficult to prove stability for the quantum systems when only one species of charged particles are fermions and the other particles have arbitrary statistics by adapting the methods of Federbush [12] or Dyson and Lenard [5] developped for the stability of matter problem in three space dimensions. It is well known that it suffices to prove stability for Yukawa forces. The difference between Coulomb- and Yukawa potential is an $H$-stable interaction in the sense of [26]. This is proved by arguments similar to the ones used in Lemma 2.1 and 4.2 .

\section{Definitions and Preliminary Results}

Let $\Lambda$ be an open region (e.g. a rectangle) in $\mathbb{R}^{2}$. We define the Hilbert space for $k$ particles of positive and $n$ particles of negative charge in the region $\Lambda$ by

$$
\mathscr{H}_{\Lambda}(k, n)=\left(\mathbb{C}^{l} \otimes L^{2}\left(\Lambda, d^{2} x\right)\right)^{\otimes_{a} k} \otimes\left(\mathbb{C}^{l} \otimes L^{2}\left(\Lambda, d^{2} x\right)\right)^{\otimes_{a^{n}}},
$$

where $\mathbb{C}^{l} \otimes L^{2}\left(\Lambda, d^{2} x\right)$ is the one particle space associated with $\Lambda-\mathbb{C}^{l}$ accounts for internal (e.g. spin) degrees of freedom - and $\otimes_{a}$ denotes the antisymmetric tensor product required for Fermi statistics. 
The kinetic energy is given by

$$
H_{\Lambda, \mu}^{0}(k, n)=-\left\{\sum_{j=1}^{k} \frac{\Delta_{x_{i}, \Lambda}}{2 M}+\sum_{i=1}^{n} \frac{\Delta_{y_{i}, \Lambda}}{2 M}\right\}+(k+n) \cdot \mu,
$$

where $M$ is the mass of the particles, $\mu \geqq 0$ is the chemical potential, and $\Delta_{\text {., } \Lambda}$ is the two dimensional Laplacian with some selfadjoint boundary conditions at $\partial \Lambda$. For the sake of concreteness we choose 0 -Dirichlet data at $\partial \Lambda$; we could instead use periodic or Neumann boundary conditions without any difficulties.

The potential energy is given by

$$
\begin{aligned}
U_{\varepsilon^{2} V_{m}}^{(k, n)}\left(X^{k}, Y^{n}\right)=\varepsilon^{2} & \sum_{1 \leqq i<j \leqq k} V_{m}\left(x_{i}-x_{j}\right)+\varepsilon^{2} \sum_{1 \leqq i<j \leqq n} V_{m}\left(y_{i}-y_{j}\right) \\
& -\varepsilon^{2} \sum_{\substack{i=1, \ldots, k \\
j=1, \ldots, n}} V_{m}\left(x_{i}-y_{j}\right),
\end{aligned}
$$

where $m \geqq 0$ and $V_{m}$ has been defined in (4.1).

If $m=0$ we require neutrality $(k=n)$.

The total Hamiltonian is

$$
H_{\Lambda, \mu}^{\varepsilon}(k, n)=H_{\Lambda, \mu}^{0}(k, n)+U_{\varepsilon^{2} V_{m}}^{(k, n)} .
$$

It is standard to prove that $H_{\Lambda, \mu}^{\varepsilon}(k, n)$ is densely defined, selfadjoint and bounded below on $\mathscr{H}_{\Lambda}(k, n)$ and $e^{-\beta H_{\Lambda, \mu}^{\varepsilon}(k, n)}$ is trace class.

The Hilbert space and the Hamiltonian for arbitrarily many particles in the region $\Lambda$ are given by

$$
\left.\begin{array}{c}
\mathscr{H}_{\Lambda}=\bigoplus_{k, n=0}^{\infty} \mathscr{H}_{\Lambda}(k, n), \text { and } \\
H_{\Lambda, \mu}^{\varepsilon}=\bigoplus_{k, n=0}^{\infty} H_{\Lambda, \mu}^{\varepsilon}(k, n), \text { respectively } .
\end{array}\right\}
$$

For the purpose of (somewhat unnecessary) mathematical rigor we also introduce a regularized Hamiltonian $H_{\Lambda, \mu}^{\varepsilon, \kappa}$ :

$$
\mathrm{H}_{\Lambda, \mu}^{\varepsilon, \kappa}=\bigoplus_{k, n=0}^{\infty} H_{\Lambda, \mu}^{\varepsilon, \kappa}(k, n),
$$

with

$$
H_{\Lambda, \mu}^{\varepsilon, \kappa}(k, n)=H_{\Lambda, \mu}^{0}(k, n)+U_{\varepsilon^{2} V_{m, \kappa}}^{(k, n)}
$$

where

$$
V_{m, \kappa}(x-y)=\int d^{2} x^{\prime} d^{2} y^{\prime} h_{\kappa}\left(x-x^{\prime}\right) V_{m}\left(x^{\prime}-y^{\prime}\right) h_{\kappa}\left(y^{\prime}-y\right),
$$

and $h_{\kappa}$ is the cutoff function defined in (2.3).

The potential $U_{\varepsilon^{2} V_{m, \kappa}}$ is bounded and stable, i.e.

$$
U_{\varepsilon^{2} V_{m, \kappa}}^{(k, n)}\left(X^{k}, Y^{n}\right) \geqq(n+k) B_{\kappa},
$$

for some $B_{\kappa}>-\infty$. 
Our goal is to prove that

$H_{\Lambda, \mu}^{\varepsilon, \kappa} \geqq-K(\varepsilon, \mu)|\Lambda|$,

for some finite constant $K(\varepsilon, \mu)$, and uniformly in $\kappa$ and $m \geqq 0$. Let $\operatorname{Tr}^{f}$ denote the trace with respect to $\mathscr{H}_{\Lambda}(k, n)$, or $\mathscr{H}_{\Lambda}$ (the superscript $f$ indicates Fermi statistics).

Lemma 5.1. The following are equivalent

(a) Given $\varepsilon$ there exists some positive $\beta_{0}$ such that

$\operatorname{Tr}^{f}\left(e^{-\beta_{0} H_{\Lambda, \mu}^{\varepsilon, \kappa}}\right) \leqq e^{K_{1}(\varepsilon, \mu)|\Lambda|}$,

uniformly in $\kappa$ and $m \geqq 0$.

(b) For arbitrary $\varepsilon$ and $\beta$

$\operatorname{Tr}^{f}\left(e^{-\beta H_{\Lambda, \mu}^{\varepsilon, \kappa}}\right) \leqq e^{K_{2}(\varepsilon, \beta, \mu)|\Lambda|}$,

uniformly in $\kappa$ and $m \geqq 0$.

(c) For arbitrary $\varepsilon$

$H_{\Lambda, \mu}^{\varepsilon, \kappa} \geqq-K(\varepsilon, \mu)|\Lambda|$,

uniformly in $\kappa$ and $m \geqq 0$.

Proof. We show: $(\mathrm{a}) \Rightarrow(\mathrm{c}) \Rightarrow(\mathrm{b}) \Rightarrow(\mathrm{a})$.

Obviously (a) is a special case of $(b)$, so that $(b) \Rightarrow(a)$ is trivial. To prove (a) $\Rightarrow$ (c) we notice that

$$
\operatorname{Tr}^{f}\left(e^{-\beta_{0} H_{A, \mu}^{\varepsilon_{i} \kappa}}\right) \geqq e^{-\beta_{0} E_{,, \mu}^{\varepsilon, \kappa}},
$$

where $E_{\Lambda, \mu}^{\varepsilon, \kappa} \equiv \inf \operatorname{spec}\left(H_{\Lambda, \mu}^{\varepsilon, \kappa}\right)$ is the groundstate energy of $H_{\Lambda, \mu}^{\varepsilon, \kappa}$.

Using (5.9) and taking logarithms we obtain

$$
\begin{aligned}
& E_{\Lambda, \mu}^{\varepsilon, \kappa} \geqq-\frac{1}{\beta_{0}} K_{1}(\varepsilon, \mu)|\Lambda|, \quad \text { i.e. } \\
& H_{\Lambda, \mu}^{\varepsilon, \kappa} \geqq-K(\varepsilon, \mu)|\Lambda|, \quad \text { for } \quad K(\varepsilon, \mu) \equiv \frac{1}{\beta_{0}} K_{1}(\varepsilon, \mu) .
\end{aligned}
$$

This proves (c).

For the proof of $(\mathrm{c}) \Rightarrow(\mathrm{b})$ we decompose $H_{\Lambda, \mu}^{\varepsilon, \kappa}$ :

$H_{\Lambda, \mu}^{\varepsilon, \kappa}=\frac{2}{3} H_{\Lambda, \mu}^{0}+\frac{1}{3} H_{\Lambda, \mu}^{\sqrt{3} \varepsilon, \kappa}$.

Applying the Golden-Thompson inequality we get

$$
\begin{aligned}
\operatorname{Tr}^{f}\left(e^{-\beta H_{A, \mu}^{\varepsilon, \kappa}}\right) & \leqq e^{\frac{\beta}{3} E_{\Lambda, \mu}^{\sqrt{3} \varepsilon, \kappa}} \operatorname{Tr}^{f}\left(e^{\left.-\frac{2}{3} \beta H_{\Lambda, \mu}^{0}\right)}\right. \\
& \leqq e^{\beta|\Lambda|\left(\frac{1}{3} K(\sqrt{3} \varepsilon, \mu)+3 c_{\beta} e^{\mu}\right)}
\end{aligned}
$$

and $c_{\beta}$ is some constant determined below. Instead of the Golden-Thompson inequality we could also use the Trotter product formula and the Hölder inequality for the trace. Q.E.D.

Next we want to verify (a) by reducing this inequality to the estimates of Sections 3 and 4 . 
Theorem 5.2. For $\beta \varepsilon^{2}<4 \pi$

$$
\operatorname{Tr}^{f}\left(e^{-\beta H_{\Lambda, \mu}^{\varepsilon_{,} \kappa}}\right) \leqq \Xi\left(\beta \varepsilon^{2}, V_{m, \kappa}, c_{\beta} e^{\mu} \chi_{\Lambda}\right)
$$

where $\Xi$ is the classical partition function in the grand canonical ensemble estimated in Sections 3 and $4, e^{\mu}$ is the fugacity, and

$$
c_{\beta}=l \cdot \int e^{-\beta \frac{p^{2}}{2 M}} d^{2} p=2 M \pi l \beta^{-1}
$$

with $l$ the number of internal degrees of freedom. (Notice that $\Xi$ depends on $\beta \varepsilon^{2}$. This dependence was suppressed in the notation of Sections 3 and 4.)

Proof. Let $P_{\beta}^{\Lambda}(x, y)$ denote the kernel of $e^{-\beta\left(-\Delta_{\Lambda} / 2 M\right)}$. By the Golden-Thompson inequality

$$
\begin{aligned}
\operatorname{Tr}^{f}\left(e^{-\beta H_{\Lambda, \mu}^{\varepsilon, \kappa}}\right) \leqq & \operatorname{Tr}^{f}\left(e^{\left.-\beta H_{\Lambda, \mu}^{0}, e^{-\beta U_{\varepsilon^{2} V_{m, \kappa}}}\right)}\right. \\
\leqq & \sum_{k, n=0}^{\infty} \operatorname{Tr}^{f}\left(e^{-\beta H_{\Lambda, \mu}^{0}(k, n)} e^{\left.-\beta U_{\varepsilon^{2} V_{m, n}}^{(k, n)}\right)}\right. \\
= & \sum_{k, n=0}^{\infty} \frac{\left(l e^{\mu}\right)^{(k+n)}}{k ! n !} \int_{\Lambda^{\times k}} d X^{k} \int_{\Lambda_{\times n}} d Y^{n} \operatorname{Det}\left(P_{\beta}^{\Lambda}\left(x_{i}, x_{j}\right)\right) \\
& \times \operatorname{Det}\left(P_{\beta}^{\Lambda}\left(y_{i}, y_{j}\right)\right) e^{-\beta U_{\varepsilon^{2} V_{m, \kappa}}^{(k, n)}\left(X^{k}, Y^{n}\right)} \\
\leqq & \sum_{k, n=0}^{\infty} \frac{\left(l e^{\mu}\right)^{(k+n)}}{k ! n !} \int_{\Lambda^{\times k}} d X^{k} \int_{\Lambda^{\times n}} d Y^{n} \prod_{i=1}^{k} P_{\beta}^{\Lambda}\left(x_{i}, x_{i}\right) \\
& \times \prod_{j=1}^{n} P_{\beta}^{\Lambda}\left(y_{j}, y_{j}\right) e^{-\beta U_{\varepsilon^{2} V_{m, \kappa}}^{(k, n)}\left(X^{k}, Y^{n}\right)},
\end{aligned}
$$

and we have used Hadamard's inequality for determinants of positive definite matrices $^{2}$.

Since we are working with the Laplacian $\Delta_{\Lambda}$ with 0 -Dirichlet data at $\partial \Lambda$,

$$
P_{\beta}^{\Lambda}(x, x)<\int e^{-\beta p^{2} / 2 M} d^{2} p=2 M \pi \beta^{-1} .
$$

(Similar, but slightly worse bounds which are independent of $\Lambda$ also hold for periodic or Neumann boundary conditions.) Therefore

$$
\begin{aligned}
\operatorname{Tr}^{f}\left(e^{\left.-\beta H_{\Lambda, \mu}^{\varepsilon, \mu}\right)}\right. & \leqq \sum_{k, n=0}^{\infty} \frac{\left(c_{\beta} e^{\mu}\right)^{(k+n)}}{k ! n !} \int_{\Lambda^{\times k}} d X^{k} \int_{\Lambda^{\times n}} d Y^{n} e^{-\beta U_{\varepsilon^{2} V_{m, \kappa}}^{(k, n)}\left(X^{k}, Y^{n}\right)} \\
& \leqq \Xi\left(\beta \varepsilon^{2}, V_{m, \kappa}, c_{\beta} e^{\mu} \chi_{\Lambda}\right)
\end{aligned}
$$

Our proof covers the case $m=0$, but then terms with $k \neq n$ vanish by Lemma 4.2, Corollary 4.3. Uniform estimates on $\Xi$ are given for $m>0$ in Corollary 3.5 and for $m=0$ in Theorem 4.4. Q.E.D.

2 I thank E. Lieb for suggesting to apply Hadamard's inequality which simplified matters considerably. 


\section{Corollary 5.3.}

(a) $\operatorname{Tr}^{f}\left(e^{-\beta H_{\Lambda, \mu}^{\varepsilon}}\right) \leqq e^{K_{2}(\varepsilon, \beta, \mu)|\Lambda|}$

(b) $\lim _{\Lambda \rightarrow \mathbb{R}^{2}} \frac{1}{|\Lambda|} \log \operatorname{Tr}^{f}\left(e^{-\beta H_{\Lambda, \mu}^{\varepsilon}}\right) \equiv p(\beta, \alpha, z)$

exists; here $z \equiv e^{\mu}$ and $\alpha \equiv \frac{\beta \varepsilon^{2}}{4 \pi}$.

Proof. (a) It suffices to prove (a) for the potential $V_{m}$ with $m>0$. For, if $m=0$ and $k=n$ (neutrality)

$$
U_{\varepsilon^{2} V_{0}}^{(n, n)}>U_{\varepsilon^{2} V_{m}}^{(n, n)} .
$$

It is easy to see that on some dense domain in $\mathscr{H}_{A}$

$$
\mathrm{S}-\lim _{\kappa \rightarrow \infty} H_{\Lambda, \mu}^{\varepsilon, \kappa}=H_{\Lambda, \mu}^{\varepsilon} .
$$

Hence $\lim _{\kappa \rightarrow \infty} E_{\Lambda, \mu}^{\varepsilon, \kappa} \leqq E_{\Lambda, \mu}^{\varepsilon} \equiv \inf \operatorname{spec}\left(H_{\Lambda, \mu}^{\varepsilon}\right)$,

i.e.

$$
H_{\Lambda, \mu}^{\varepsilon} \geqq E_{\Lambda, \mu}^{\varepsilon} \geqq-K(\varepsilon, \mu)|\Lambda|,
$$

by Lemma $5.1((\mathrm{a}) \Rightarrow(\mathrm{c})$ ), Theorem 5.2, Corollary 3.5 (and Theorem 4.4). For fixed $m>0$, arbitrary finite $k$ and $n$, a bounded region $\Lambda$ and arbitrary $\lambda>0$

$$
\begin{aligned}
& \left\|U_{3 \varepsilon^{2} V_{m}}^{(k, n)}\left(\lambda \cdot H_{\lambda, \mu}^{0}(k, n)+K_{\lambda}\right)^{-1}\right\| \\
& \leqq \frac{k+n}{2}(k+n+1) 3 \varepsilon^{2} \int d^{2} p\left[\left(p^{2}+m^{2}\right)^{-1}+\frac{1}{4 \pi}\left|\ln \left(c m^{2}\right)\right| \delta^{(2)}(p)\right]\left(\lambda p^{2} / 2 M+K_{\lambda}\right)^{-1}
\end{aligned}
$$

$<1$, provided we choose $K_{\lambda}$ large enough.

It is well known that this estimate implies

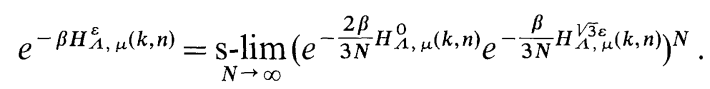

It is easy to show that this equation and the Hölder inequality for $\operatorname{Tr}^{f}$ yield

$$
\operatorname{Tr}^{f}\left(e^{-\beta H_{\Lambda, \mu}^{\varepsilon}(k, n)}\right) \leqq\left\|e^{-\frac{\beta}{3} H_{\Lambda, \mu}^{\sqrt{3} \varepsilon}(k, n)}\right\| \cdot \operatorname{Tr}^{f}\left(e^{-\frac{2 \beta}{3} H_{\Lambda, \mu}^{0}(k, n)}\right) .
$$

By (5.12)

$$
\left\|e^{-\frac{\beta}{3} H_{A, \mu}^{\sqrt{3} \varepsilon}(k, n)}\right\| \leqq\left\|e^{-\frac{\beta}{3} H_{A, \mu}^{\sqrt{3} \varepsilon}}\right\| \leqq e^{\frac{\beta}{3} K(\sqrt{3} \varepsilon, \mu)|\Lambda|} .
$$

Summing (5.15) over all $k$ and $n$ we therefore obtain

$$
\operatorname{Tr}^{f}\left(e^{-\beta H_{\Lambda, \mu}^{\varepsilon}}\right)<e^{\beta|\Lambda|\left(\frac{1}{3} K(\sqrt{3} \varepsilon, \mu)+3 c_{\beta} e^{\mu}\right)},
$$

completing the proof of (a).

(b) For the proof of (b) we refer the reader to the methods of [26] and [19] which apply to our case with minor changes. Q.E.D. 
Remarks. 1) Using estimates (5.13) and equation (5.14) a direct proof of the Golden-Thompson inequality in the limit $\kappa=\infty$ and for $m \geqq 0$ can be given:

$$
\operatorname{Tr}^{f}\left(e^{-\beta H_{\Lambda, \mu}^{\varepsilon}(k, n)}\right) \leqq \operatorname{Tr}^{f}\left(e^{-\beta H_{\Lambda, \mu}^{0}(k, n)} e^{-\beta U_{\varepsilon^{2} V_{m}}^{(k, n)}}\right) .
$$

Let $m=0$ and $k=n$. Since $\Lambda$ is a bounded set

$$
\left|V_{0}(x)-V_{1}(x)\right|<K_{\Lambda}, \text { for all } x \in \Lambda,
$$

i.e.

$$
\left\|\left(V_{0}-V_{1}\right) \uparrow L^{2}\left(\Lambda, d^{2} x\right)\right\|<K_{\Lambda},
$$

for some finite constant $K_{\Lambda}$.

Therefore estimate (5.13) and hence (5.14) extend to the case where $m=0$ and $k=n$ yielding a direct proof of the Golden-Thompson inequality for $\kappa=\infty$, $m=0, k=n$.

Let $\beta \varepsilon^{2}<4 \pi$. Applying then the Golden-Thompson inequality and repeating the arguments given in the proof of Theorem 5.2 we get (using Corollary 3.5 and Theorem 4.4)

$$
\operatorname{Tr}^{f}\left(e^{-\beta H_{\Lambda}^{\varepsilon}, \mu}\right) \leqq \Xi\left(\beta \varepsilon^{2}, V_{m}, c_{\beta} e^{\mu} \chi_{\Lambda}\right),
$$

for arbitrary $m \geqq 0$.

Let $p_{\mathrm{cl}}$. denote the pressure of the classical gas (including the contribution of the kinetic energy) and $p_{\text {qu. }}$ the pressure of the quantum Fermi gas. We derive from (5.16) by taking logarithms that

$$
p_{\text {cl. }} \geqq p_{\text {qu. }}{ }^{3} \text {. }
$$

2) For $m>0, \beta \varepsilon^{2}<4 \pi$ and large $\mu$ it seems to be rather straightforward to apply the Glimm-Jaffe-Spencer cluster expansion [7] with modifications due to Federbush [12] to construct the Euclidean (temperature-ordered) Green's functions for the quantum Yukawa gas. If this is true we may apply a theorem proved in [11] (and refs. given there) to conclude

Quasi-Theorem. For $m>0, \beta \varepsilon^{2}<4 \pi$ and $\mu$ large enough the Euclidean Green's functions for the two component quantum Yukawa gas with Fermi statistics exist, are holomorphic in the time differences on a tubular domain - see [11] and refs. given there - and have tempered boundary values on the real time region satisfying the KMS condition.

This will possibly be proved in a future publication.

3) Sure enough the two component quantum Coulomb gas has scaling properties, too:

Using the obvious scaling properties of the Laplacian and the existence of the thermodynamic limit (Corollary 5.3, (b)) we may prove as in Section 4, (c)

$$
\lambda^{2} p(\beta, \alpha, z)=p\left(\lambda^{-2} \beta, \alpha, \lambda^{2-\alpha} z\right),
$$

whence

$$
p(\beta, \alpha, z)=z^{\frac{2}{2-\alpha}} F\left(\alpha, \beta \cdot z^{\frac{2}{2-\alpha}}\right) .
$$

3 I thank I. Herbst for pointing out to me (5.17) in a slightly different context. 


\section{References}

1. Albeverio, S., Høegh-Krohn, R.: Commun. math. Phys. 30, 171 (1973)

2. Coleman, S.: Quantum Sine-Gordon Equation as the Massive Thirring Model, to appear in Phys. Rev. D.

3. Dashen, R., Hasslacher, B., Neveu,A.: The Particle Spectrum in Model Field Theories from Semi-Classical Functional Integral Techniques, Inst. Adv. Study, Preprint (1975)

4. Deutsch, C., Lavaud, M.: Phys. Rev. A 9, 2598 (1974)

5. Dyson, F., Lenard, A.: J. Math. Phys. 8, 423 (1967); J. Math. Phys. 9, 698 (1968)

6. Edwards, S., Lenard, A.: J. Math. Phys. 3, 778 (1962)

7. Erice Summer School in: Constructive Quantum Field Theory, G. Velo, A. Wightman (eds.), Lecture Notes in Physics 25, Berlin-Heidelberg-New York: Springer 1973

8. Fröhlich, J.: Phys. Rev. Letters 34, 833 (1975), and paper in preparation

9. Fröhlich,J., Seiler,E.: The Massive Thirring-Schwinger Model as the Massive Sine-Gordon Equation: Convergence of Perturbation Theory, Harvard, Preprint (1975)

10. Fröhlich, J.: Schwinger Functions and Their Generating Functionals, II..., to appear in Adv. Math.

11. Fröhlich, J.: The Pure Phases, the Irreducible Quantum Fields..., Princeton University, Preprint (1975); The Reconstruction of Quantum Fields... at Arbitrary Temperatures, to appear in Helv. Phys. Acta 1975

12. Federbusch, P.: J. Math. Phys. 16, 347 (1975); 16, 706 (1975)

13. Ginibre, J.: Commun. math. Phys. 16, 310 (1970)

14. Glimm,J., Jaffe,A.: In: Mathematics of Contemporary Physics, R.F.Streater (ed.), p. 77. London-New York: Academic Press (1972)

15. Guerra, F.: Phys. Rev. Letters 28, 1213 (1972)

16. Guerra,F., Rosen, L., Simon, B.: The $P(\phi)_{2}$ Euclidean Quantum Field Theory as Classical Statistical Mechanics, to appear in Ann. Math.

17. Knorr, G.: Phys. Letters 28A, 166 (1968)

18. Lieb, E., Mattis, D.: J. Math. Phys. 6, 304 (1965)

19. Lieb, E., Lebowitz, J.: Adv. Math. 9, 316 (1972)

20. Luther,A., Emery, V. J.: Phys. Rev. Letters 33, 589 (1974)

21. May, R.: Phys. Letters 25A, 282 (1967)

22. Minlos, R.: Tr. Mosk. Math. Obs. 8, 471 (1959)

23. Nelson, E.: J. Funct. Anal. 12, 211 (1973)

24. Nelson, E.: In: Partial Differential Equations, D. Spencer (Ed.), Symp. Pure Math. 23, A.M.S. Publications, 1973, p. 413

25. Nelson, E.: contribution in ref. 7

26. Ruelle, D.: Statistical Mechanics. New York: Benjamin (1969)

27. Schwinger, J.: Phys. Rev. 128, 2425 (1962)

28. Simon, B.: The $P(\phi)_{2}$ Euclidean (Quantum) Field Theory. Princeton Series in Physics. Princeton: Princeton University Press 1974

29. Wightman,A.S.: In: Cargèse Lectures in Theoretical Physics. New York-London-Paris: Gordon \& Breach 1967

Communicated by J. L. Lebowitz

Received July 16, 1975 